\title{
Effects of Shizhifang on NLRP3 Inflammasome Activation and Renal Tubular Injury in Hyperuricemic Rats
}

\author{
Yansheng Wu, ${ }^{1}$ Fei He, ${ }^{1,2}$ Yingqiao Li, ${ }^{1,3}$ Huiling Wang, ${ }^{1}$ Liqiang Shi, ${ }^{1}$ \\ Qiang Wan, ${ }^{1}$ Jiaoying Ou, ${ }^{1,4}$ Xiaoying Zhang, ${ }^{5}$ Di Huang, ${ }^{1}$ Lin Xu, ${ }^{1}$ Pinglan Lin, \\ Guanghui Yang, ${ }^{6}$ Liqun He, ${ }^{1}$ and Jiandong Gao ${ }^{1}$
}

\author{
${ }^{1}$ Department of Nephrology, Shuguang Hospital Affiliated to Shanghai University of Traditional Chinese Medicine, \\ TCM Institute of Kidney Disease of Shanghai University of Traditional Chinese Medicine, Shanghai Key Laboratory of Traditional \\ Chinese Clinical Medicine, No. 528 Zhangheng Road, Shanghai 201203, China \\ ${ }^{2}$ Department of Nephrology, Xiamen Hospital of Traditional Chinese Medicine, No. 1739 Xianyue Road, Xiamen 361009, China \\ ${ }^{3}$ Department of Nephrology, Traditional Chinese Medicine Hospital of Langfang City, No. 108 North Yinhe Road, \\ Langfang 065000, China \\ ${ }^{4}$ Department of Internal Medicine, Shanghai TCM-Integrated Hospital Affiliated to Shanghai University of Traditional \\ Chinese Medicine, No. 184 Baoding Road, Shanghai 200082, China \\ ${ }^{5}$ Department of Laboratory, Shuguang Hospital Affiliated to Shanghai University of Traditional Chinese Medicine, \\ No. 528 Zhangheng Road, Shanghai 201203, China \\ ${ }^{6}$ Department of Rheumatology and Immunology, Shuguang Hospital Affiliated to Shanghai University of Traditional Chinese Medicine, \\ No. 528 Zhangheng Road, Shanghai 201203, China
}

Correspondence should be addressed to Liqun He; heliqun59@163.com and Jiandong Gao; gaojiandong@hotmail.com

Received 13 May 2017; Revised 25 July 2017; Accepted 16 August 2017; Published 12 November 2017

Academic Editor: Jae Youl Cho

Copyright (C) 2017 Yansheng Wu et al. This is an open access article distributed under the Creative Commons Attribution License, which permits unrestricted use, distribution, and reproduction in any medium, provided the original work is properly cited.

\begin{abstract}
Objective. Uric acid (UA) activates the NLRP3-ASC-caspase-1 axis and triggers cascade inflammatory that leads to hyperuricemic nephropathy and hyperuricemia-induced renal tubular injury. The original study aims to verify the positive effects of the traditional Chinese medicinal formula Shizhifang (SZF) on ameliorating the hyperuricemia, tubular injury, and inflammasome infiltration in the kidneys of hyperuricemic lab rats. Method. Twenty-eight male Sprague-Dawley rats were divided into four groups: control group, oxonic acid potassium (OA) model group, OA + SZF group, and OA + Allopurinol group. We evaluated the mediating effects of SZF on renal mitochondrial reactive oxygen species (ROS) and oxidative stress (OS) products, protein expression of NLRP3ASC-caspase-1 axis, and downstream inflammatory factors IL-1 $\beta$ and IL-18 after 7 weeks of animals feeding. Result. SZF alleviated OA-induced hyperuricemia and inhibited OS in hyperuricemic rats $(P<0.05)$. SZF effectively suppressed the expression of gene and protein of the NLRP3-ASC-caspase-1 axis through accommodating the ROS-TXNIP pathway $(P<0.05)$. Conclusion. Our data suggest that SZF alleviates renal tubular injury and inflammation infiltration by inhibiting NLRP3 inflammasome activation triggered by mitochondrial ROS in the kidneys of hyperuricemic lab rats.
\end{abstract}

\section{Introduction}

Economic development has led to marked changes in eating habits. The increased intake of fat, salt, and calories has elevated the prevalence of hyperuricemia and hyperuricemic nephropathy, leading to an increase in preventative research in the field of kidney health. Extensive epidemiological surveys and experimental research outcomes have revealed that hyperuricemia is closely associated with gout [1], diabetes $[2,3]$, metabolic syndrome [4], chronic heart failure [5], and nervous system disease $[6,7]$. A growing body of evidence suggests that slightly elevated uric acid (UA) could lead to 
hypertension, retinal vascular disease, and renal injury. In Asian countries, $26.1 \%$ of men and $17.1 \%$ of women exhibit slightly elevated UA [8]. One survey [9] found that 120 million people in China have received diagnoses of hyperuricemia and that the age of onset is decreasing, with regions such as Shandong province achieving a hyperuricemia morbidity rate of $13.19 \%$. The results of a recent cross-sectional study [10] validated an independent correlation between hyperuricemia and a reduced glomerular filtration rate in patients with CKD. Another study [11] showed that the prevalence of hyperuricemia in patients diagnosed with Stage 1-3 CKD is between $40 \%$ and $60 \%$ and that in patients with Stage 4 or 5 CKD is $70 \%$. Therefore, UA levels have become a predictive factor for evaluating CKD development and subsequent renal inflammation has become a key factor in CKD progressing to end-stage renal disease (ESRD). Hence, the research and prevention of hyperuricemia have become global endeavours.

UA plays a proinflammatory role in numerous signalling pathways. Recent studies $[12,13]$ have found that soluble UA and UA crystals activate the NLRP3 (NLR family pyrin domain containing 3 ) inflammasome, causing proinflammatory cytokines such as IL- $1 \beta$ to mature and trigger congenital immune defence against danger signals such as infection and metabolic disorder. The NLRP3 inflammasome is a type of polyprotein complex formed through the interconnection and oligomerisation of NLRP3 receptor proteins, apoptosisassociated speck-like proteins containing a caspase recruitment domain (ASC), and caspase-1. NLRP3 receptor proteins can identify pathogen-associated molecular patterns and damage-associated molecular patterns (DAMPs). DAMPs are found in various components of the body, such as damaged tissues and substances released after tissue necrosis (e.g., adenosine triphosphate [ATP] or UA) [14]. Currently, NLRP3 receptor proteins are believed to be activated by the efflux of potassium ions, generated reactive oxygen species (ROS), or controlled by tissue protease released by ruptured lysosomes. Among these mechanisms, the most widely accepted for activation of NLRP3 is mitochondrial ROS generation [15]. Zhou et al. [16] observed an unexpected pivotal function in mitochondria during ROS generation. Specifically, the NLRP3 inflammasome is excited by ROS secreted by damaged mitochondria. These findings suggest that mitochondria are essential for both apoptosis and inflammatory responses. The release of ROS causes thioredoxin interacting proteins (TXNIP) to separate from oxidised thioredoxin-1 (Trx-1) proteins and bond with the leucine-rich repeat domain of NLRP3 receptor proteins, which consequently activates the NLRP3 inflammasome [17]. Therefore, the ROS-TXNIP pathway becomes a precondition for activation of the NLRP3-ASCcaspase-1 inflammatory pathway. Recent research [18] has shown that once the NLRP3-ASC-caspase-1 axis is activated, the inflammatory response mediated by caspase-1 triggers a type of programmed cell death: pyroptosis. Pyroptosis may be a newly identified mechanism for UA-induced renal inflammation and injury.

SZF is a traditional Chinese medicinal formula comprising plantago seeds, white mustard seeds, vaccaria seeds, and abutilon seeds. This formula, patented in China, has been used for over 20 years in the clinical treatment of hyperuricemia and hyperuricemic nephropathy, and the results have been reliable $[19,20]$. To empirically elucidate the mechanisms underlying the effects of SZF on renal function, we used SZF to treat rats with mild hyperuricemia and then examined the interference of SZF on the NLRP3 signalling pathways in their kidneys. Based on this intervention, we hypothesised that SZF suppresses the renal ROS generation of the hyperuricemic lab rats, thereby inhibiting renal and oxidative stress (OS), obstructing the assembly of the NLRP3 inflammasome and activation of the NLRP3-ASC-caspase-1 axis, and reducing tubular inflammatory responses. Moreover, SZF may reduce renal cell pyroptosis and protect tubular cell function.

\section{Materials and Methods}

2.1. Animals. Twenty-eight healthy adult male SpragueDawley rats (SPF-grade) weighing $200 \pm 20$ g were examined. The rats were provided by the Shanghai Laboratory Animal Centre (SLAC). This study was conducted in accordance with safe animal testing specifications (Safety Certificate Number: SYXK-HU-2008-0016, Animal Ethics Code SZY201610008). The rats were kept at the Shanghai University of Traditional Chinese Medicine Animal Testing Centre at $25^{\circ} \mathrm{C}$ (relative humidity, $45 \%$ ) with a 12-hour day/night cycle under artificial light. They were given ad libitum access to water. After an adaptation period of 1 week, we commenced with the proposed diet and interventional treatment. All animal tests were approved by the Shanghai University of Traditional Chinese Medicine Animal Testing Centre.

2.2. Reagents and Drugs. Oxonic acid potassium salt (OA, 156124) was purchased from Sigma-Aldrich (United States,) and standard feed containing $2 \%$ OA was prepared by the SLAC. SZF dry powder made by Shanghai Chinese Traditional Pharmaceutical Technology Co., Ltd. (China), was dissolved in distilled water to produce the equivalent of the crude drug concentration of $2.68 \mathrm{~g} / \mathrm{kg}$ and was administered intragastrically. Allopurinol tablets $(0.1 \mathrm{~g}, 121203)$ were purchased from Beijing Yanjing Pharmaceutical Co., Ltd. The UA testing kit (C012-1), creatinine testing kit (C011-2), urea nitrogen testing kit (C013-2), mitochondrial sampling and testing kit (G006), glutathione peroxidase testing kit (GSH-PX A005), MDA testing kit (A003-1), superoxide dismutase (SOD) testing kit (A001-3), and CAT testing kit (A007-1) were provided by Nanjing Jiancheng Bioengineering Institute (Nanjing, China). The enzyme-linked immunosorbent assay (ELISA) for rat ROS (DRE901Ra) was provided by AMEKO (China). The rat IL-18 (SU-B30650) and IL$1 \beta$ (SU-B30419) ELISA kit were purchased from Kenuodi (Quanzhou, China) Biological Technology Co., Ltd. Rabbit anti-rat IL-18 (H-173) and ASC (N-15-R) antibodies were purchased from Santa Cruz Biotechnology (United States). Rabbit anti-rat 3-NT (APG8A) antibodies were purchased from Biovision (United States). Rabbit anti-rat IgG IL-1 $\beta$ (ab9787), caspase-1 (ab10862), TXNIP (ab86983), and 4-HNE (ab46545) were purchased from Abcam (British). Rabbit antirat IgG NLRP3 (NALP3; Nbp2-12446) was purchased from 
NOVUS (United States). The ready-to-use rabbit IgG-SABC immunohistochemical and SABC-AP (mice IgG) testing kits and $\mathrm{DAB}$ colour development kit were purchased from Boster (Wuhan, China). GAPDH antibodies (5174S) were purchased from Cell Signalling Technology (United States). The HRP secondary antibodies were provided by Jackson ImmunoResearch Laboratories. The mRNA sampling and testing kit was purchased from Tiangen Biotech (Beijing, China) Co., Ltd. The reverse transcription kit (RR036A) and SYBR Premix Ex Taq TM testing kit (RR420A) were provided by Takara Biotechnology Co., Ltd. (Japan).

2.3. SZF Fingerprint Identification. Shanghai Chinese Traditional Pharmaceutical Technology Co., Ltd. (China), was commissioned to perform fingerprint identification on the SZF. Vaccaria tablets (30 g; batch number 140220), white mustard seed tablets ( $30 \mathrm{~g}$; batch number LY1505016), abutilon seed tablets ( $30 \mathrm{~g}$; batch number $160517 \mathrm{HY})$, and plantago seed tablets (60 g; batch number 151225) were obtained from Shanghai Union Dispensary Co. (China). The origins of the medicinal herbs used to produce these tablets were, respectively, Jilin, Anhui, Jiangxi, and Anhui provinces, China. The tablets were blended, crushed, and placed in herb bags according to the SZF formula. Each bag (one unit) was submerged in eight units of water for 1 hour and then heated under reflux twice for 1 hour each. The filtrates were combined and blended to produce the test solution. The test solution was analysed against several control samples (i.e., geniposidic acid, calycosin-7-glucoside, vaccaria-glucoside, and sinapine thiocyanate) and a blank (water) to produce the liquid fingerprints of the test solution. Water extraction was performed on the four medicinal herbs (i.e., fried plantago seeds, fried vaccaria, white mustard seeds, and abutilon seeds). A liquid analysis was then conducted on the extracts. Each extract was sampled six times to calculate the relative retention time and relative standard deviation of the relative peak area. The results showed that all extracts met the test requirements. Fingerprint similarity calculation software was employed to measure the extracts. The similarity of all six extracts was $100 \%$, showing that the precision of the proposed method met the test requirements. We also observed the injection times $(0,2,4,6,8$, and $24 \mathrm{~h})$ of the six extracts. The results indicated that all samples maintained excellent stability within $24 \mathrm{~h}$. Fingerprint similarity calculations showed that the similarity of the six extracts was $99.9 \%$, verifying that the reproducibility of the proposed method met the test requirements (Figures 1(a)-1(c)).

2.4. Establishing the Hyperuricemic Rat Model and Dosage. The 28 male rats were randomly divided into four groups which were named Control group, OA group, OA + Allopurinol group, and OA + SZF group based on weight. Each group contained seven rats. From Day 1, the rats in Control group were fed on a standard diet and the rest of rats were fed on a diet containing $2 \%$ OA to produce hyperuricemic model. All rats were given ad libitum access to water, and their diet was maintained for 7 weeks. The two treatment groups underwent their respective interventions from Day 1. The dosage for the SZF was based on the standards proposed by the Administration of Substances to Laboratory Animals (10 times the adult dosage per kilogram body weight). Allopurinol was dissolved in the drinking water of the OA + Allopurinol group (concentration, $150 \mathrm{mg} / \mathrm{L}$ ). The control and OA groups were given equivalent amounts of distilled water. The intragastric volume was controlled at $2 \mathrm{ml} / \mathrm{d}$ for 7 weeks.

2.5. Sample Collection. The body weights and serum uric acid (Sua) levels of the rats were recorded before and after OA induction and at 2-week intervals. Blood samples were collected from the tail vein, left to clot in preiced tubes, and then centrifuged at $3000 \mathrm{~g}$ at $4^{\circ} \mathrm{C}$ for $5 \mathrm{~min}$ to obtain the serum. Urine samples were collected with metabolic cages at 2-week intervals during drug treatment and centrifuged at $3000 \mathrm{~g}$ for $5 \mathrm{~min}$ to remove impurities. The samples were stored at $-20^{\circ} \mathrm{C}$ for the assays. Serum and urine UA, serum creatinine, and blood urea nitrogen levels were detected using specific commercial kits purchased from Nanjing Jiancheng Bioengineering Institute (Nanjing, China). Twenty-four-hour urine uric acid (Uua) was calculated using the following formula: Uua $=$ urine UA concentration $(\mu \mathrm{mol} / \mathrm{L}) \times$ urine volume (L) in $24 \mathrm{~h}$. At the end of the drug intervention, all animals were deprived of food but not water until the following morning. Rats were anaesthetised with pentobarbital sodium $(30 \mathrm{mg} / \mathrm{kg}$ i.p.) and blood samples were collected through the aorta abdominalis. The kidney was quickly dissected. Partial cortex tissues from the left kidney were immediately frozen in liquid nitrogen. Total cellular protein and RNA were extracted from kidney tissues. These samples were stored at $-80^{\circ} \mathrm{C}$ until they were assayed. The separated serum and other partial left kidney tissues were stored at $-80^{\circ} \mathrm{C}$ for measurement of the MDA, GSH-PX, SOD, CAT, and ROS levels.

2.6. Histopathology. The right kidney tissue was fixed for 1 day at room temperature in $90 \%$ ethanol. Renal biopsies were dehydrated with a graded series of alcohol and embedded in paraffin. Specimens were cut into $3-\mu \mathrm{m}$ thick sections on a rotary microtome and mounted on APES-coated glass slides. Each section was deparaffinised in xylene, rehydrated in decreasing concentrations of alcohol in water, and stained with HE, PAS, and Masson reagents sequentially.

2.7. Mitochondrial Extraction and OS Analysis. ROS were detected in the serum and kidney tissues by using an ELISA kit according to the manufacturer's protocol. The mitochondria from the cortex tissues were then extracted using a mitochondrial isolation kit (Nanjing Jiancheng Bioengineering Institute, Nanjing, China) according to the manufacturer's protocol. SOD, CAT, GSH-PX activity, and MDA levels in the kidney cortex tissue samples were measured using commercially available kits according to the manufacturer's instructions.

2.8. Immunohistochemistry. Rat kidney cortex tissues were fixed with $90 \%$ ethanol, embedded in paraffin, and sectioned transversely. Paraffin-embedded samples were deparaffinised prior to incubation with primary antibodies at $4^{\circ} \mathrm{C}$ overnight. 
19-Oct-2016 12:36:24

3: Diode Array 254

Range: $3.986 e-1$

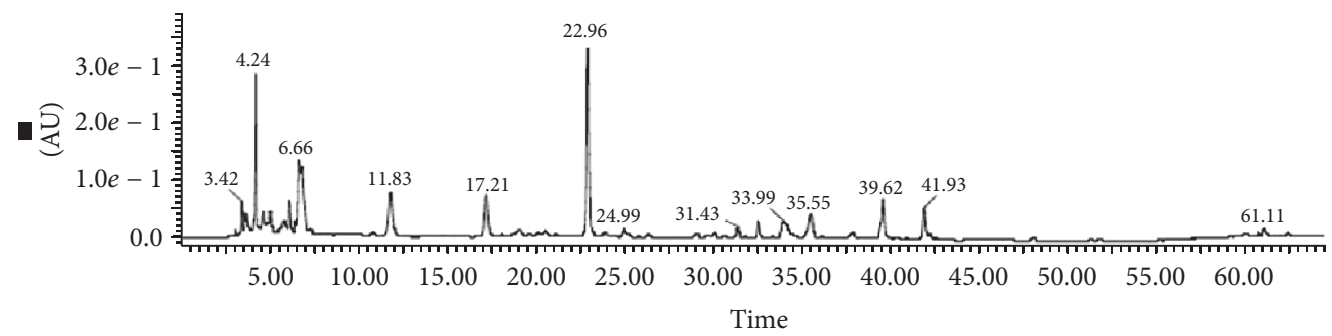

SHIZHIFANG YANGPIN-2

2: Scan ES-

TIC

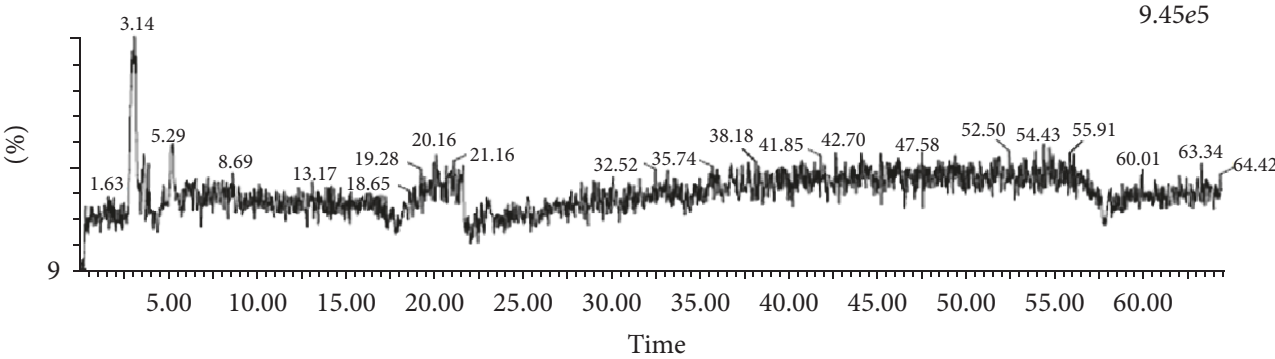

SHIZHIFANG YANGPIN-2

1: Scan ES+

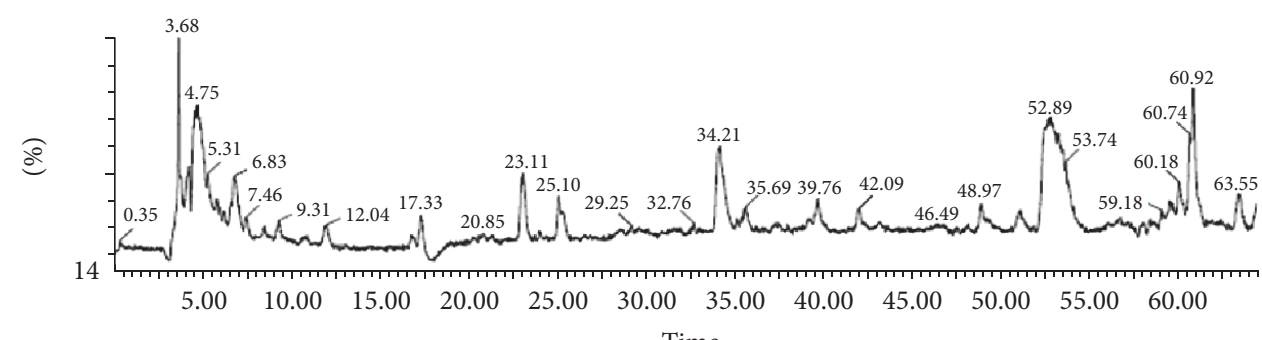

19-Oct-2016 12:36:24

SHIZHIFANG YANGPIN-2

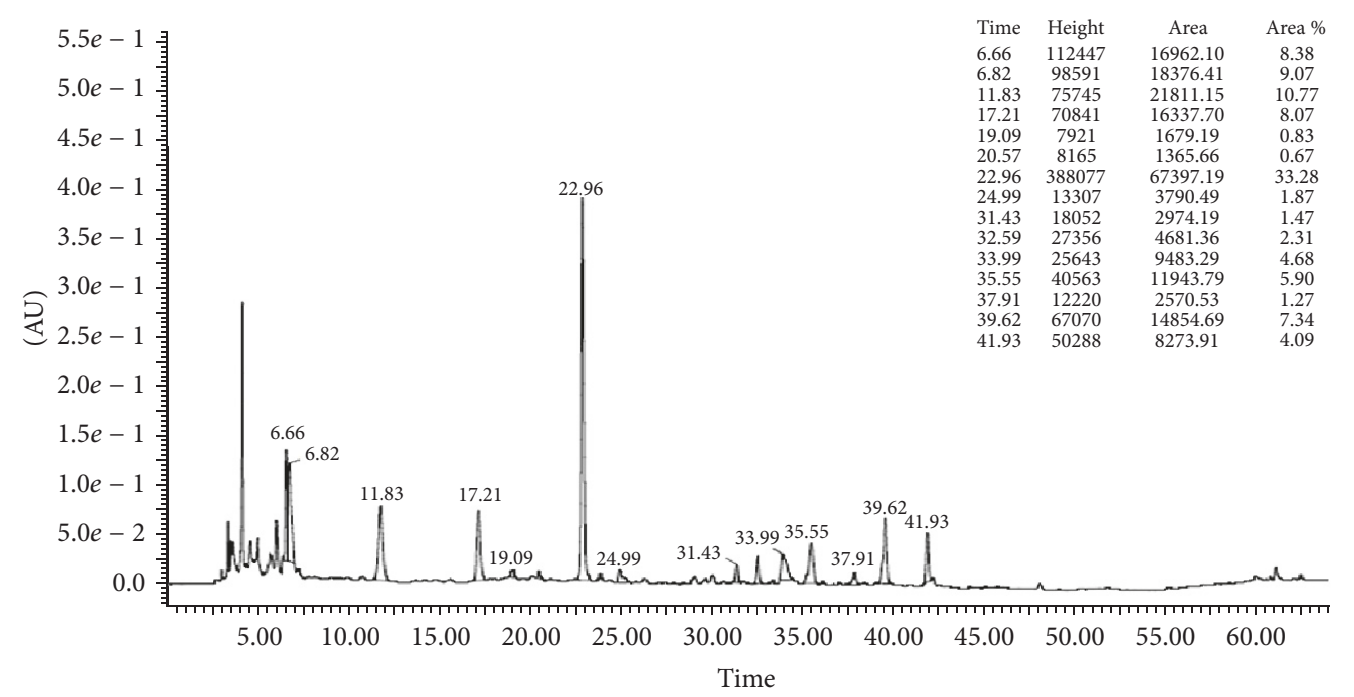

(a)

3: Diode Array

$$
254
$$

Range: $3.986 e-1$

$\begin{array}{lccc}\text { Time } & \text { Height } & \text { Area } & \text { Area \% } \\ 6.66 & 112447 & 16962.10 & 8.38 \\ 6.82 & 98591 & 18376.41 & 9.07 \\ 11.83 & 75745 & 21811.15 & 10.77 \\ 17.21 & 70841 & 16337.70 & 8.07 \\ 19.09 & 7921 & 1679.19 & 0.83 \\ 20.57 & 8165 & 1365.66 & 0.67 \\ 22.96 & 388077 & 67397.19 & 33.28 \\ 24.99 & 13307 & 3790.49 & 1.87 \\ 31.43 & 18052 & 2974.19 & 1.47 \\ 32.59 & 27356 & 4681.36 & 2.31 \\ 33.99 & 25643 & 9483.29 & 4.68 \\ 35.55 & 40563 & 11943.79 & 5.90 \\ 37.91 & 12220 & 2570.53 & 1.27 \\ 39.62 & 67070 & 14854.69 & 7.34 \\ 41.93 & 50288 & 8273.91 & 4.09\end{array}$

FIgUre 1: Continued. 

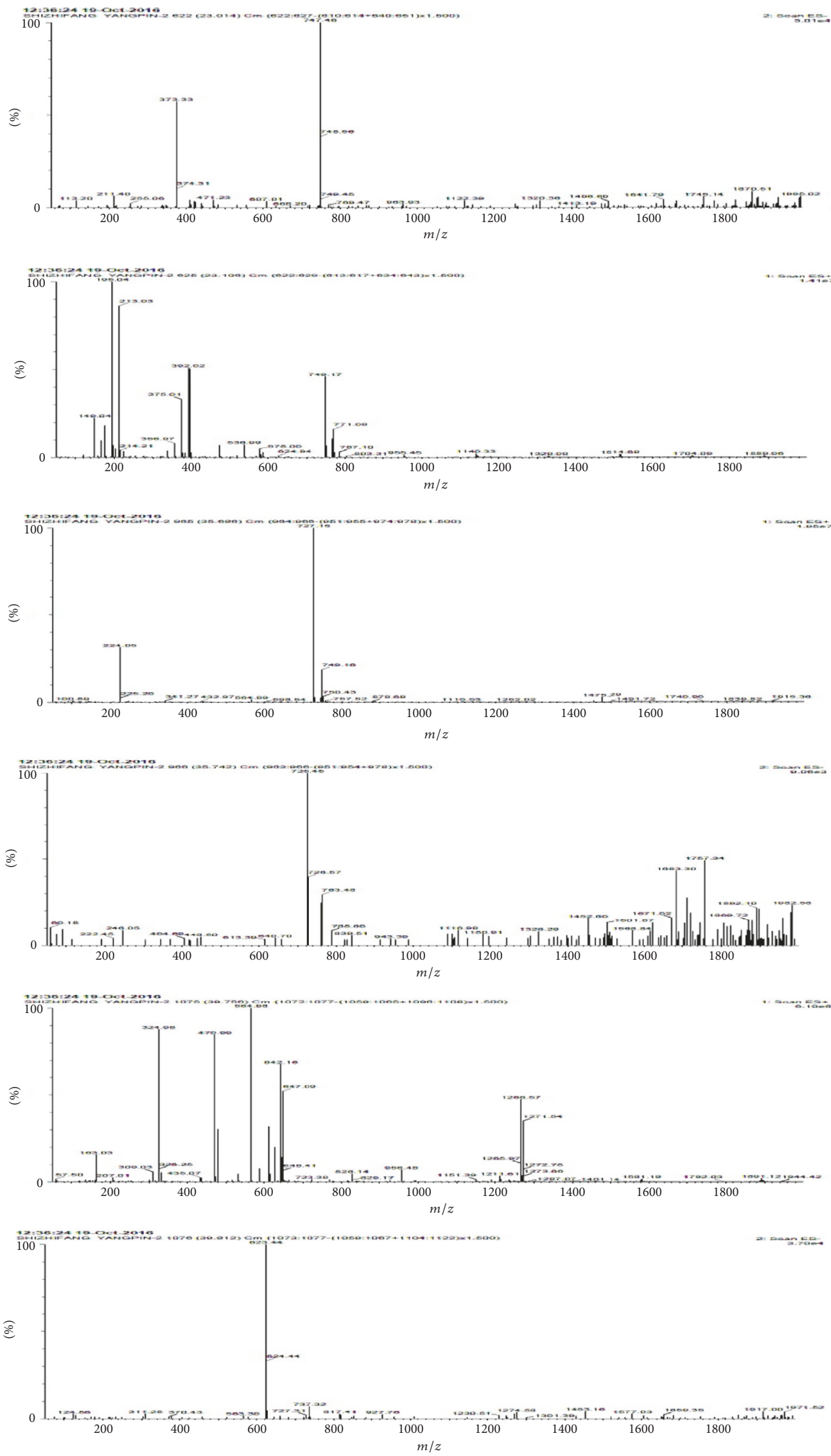

(b)

FIGURE 1: Continued. 
<smiles>O=C(O)C1=CO[C@H](O[C@@H]2O[C@H](CO)[C@@H](O)[C@H](O)[C@H]2O)[C@H]2C(CO)=CC[C@H]12</smiles>
Geniposidic acid<smiles>COc1cc(/C=C/C(=O)OCC[N+](C)(C)C)cc(OC)c1OC</smiles>

Sinapine thiocyanate<smiles>Nc1ncnc2c1ncn2C(O)OC(O)CO</smiles>

Adenosine<smiles>O=c1cc(-c2ccc(OC3OC(CO)[C@H](O)[C@H](O)[C@H]3O)cc2)oc2cc(O)c(C3O[C@H](CO)[C@@H](O)[C@H](O)C3O[C@@H]3OC[C@@H](O)[C@H](O)[C@H]3O)c(O)c12</smiles>

Vaccarin

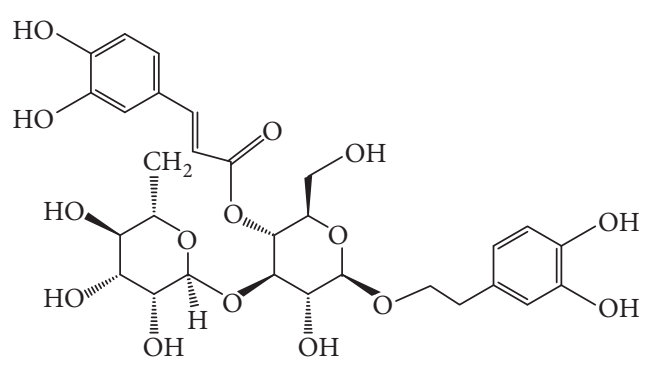

Verbascoside<smiles>COc1cc(/C=C/C(=O)O)cc(OC)c1O</smiles>

Mustard acid

(c)

FIGURE 1: Results of SZF (Shizhifang) fingerprint identification. (a) Total ion chromatography of SZF. (b) Ion flow diagram corresponding with primary ingredients of geniposidic acid, calycosin-7-glucoside, vaccaria-glucoside, and sinapine thiocyanate, obtained through LC-MS analysis. (c) Main composition of SZF.

TABLE 1: PCR primer sequence.

\begin{tabular}{|c|c|c|}
\hline Gene name & Primer sequence $\left(5^{\prime}-3^{\prime}\right)$ & $\operatorname{Tm}\left({ }^{\circ} \mathrm{C}\right)$ \\
\hline \multirow{2}{*}{ TXNIP } & F 5' GGCTTTTCTCGATCGTCCCA 3' & 60.11 \\
\hline & R 5 $5^{\prime}$ AGAGGCAGAAAGCGTTGAGT $3^{\prime}$ & 59.61 \\
\hline \multirow{2}{*}{ NLRP3 } & F $5^{\prime}$ ACGGCAAGTTCGAAAAAGGC $3^{\prime}$ & 59.97 \\
\hline & R 5' AGACCTCGGCAGAAGCTAGA $3^{\prime}$ & 60.03 \\
\hline \multirow{2}{*}{ ASC } & F $5^{\prime}$ AGACATGGGCATACAGGAGC $3^{\prime}$ & 59.53 \\
\hline & R 5' GCAATGAGTGCTTGCCTGTG 3' & 60.39 \\
\hline \multirow{2}{*}{ Caspase-1 } & F 5' AAGAAGGTGGCGCATTTCCT3' & 60.25 \\
\hline & R 5' GACGTGTACGAGTGGGTGTT3' & 59.97 \\
\hline \multirow{2}{*}{ IL-1 $\beta$} & F $5^{\prime}$ AGGATTGCTTCCAAGCCCTTGACT $3^{\prime}$ & 64.44 \\
\hline & R $5^{\prime}$ ACAGCTTCTCCACAGCCACCATGA $3^{\prime}$ & 66.22 \\
\hline \multirow{2}{*}{ IL-18 } & F $5^{\prime}$ ACAAAAGAAACCCGCCTGTG 3' & 59.26 \\
\hline & R 5' TGTGTCCTGGCACACGTTTC 3' & 61.09 \\
\hline \multirow{2}{*}{ GAPDH } & F $5^{\prime}$ TGTGAACGGATTTGGCCGTA3' & 59.96 \\
\hline & R 5 $5^{\prime}$ GATGGTGATGGGTTTCCCGT 3' & 60.03 \\
\hline
\end{tabular}

HRP-conjugated secondary antibodies were incubated for $60 \mathrm{~min}$ at room temperature. Sections were examined using a microscope (Eclipse 80i, Nikon, Japan). The antibodies used for immunohistochemistry were anti-3-NT (1:100), anti4HNE (1:100), anti-NLRP3 (1:50), anti-ASC $(1: 200)$, and anti-caspase-1 $(1: 100)$.

2.9. Real-Time PCR Analysis. Total RNA was isolated from individual rat kidneys with commercial kits for evaluating mRNA expression of NLRP3 inflammasome axis-related genes (rTXNIP, rNLRP3, rASC, rCaspase-1, rIL-1 $\beta$, rIL-18) and glyceraldehyde 3-phosphate dehydrogenase (rGAPDH). Reverse-transcribed cDNA was obtained using the SuperScript First-Strand Synthesis kit (Takara). The primers used are summarised in Table 1. All primer sequences were checked in GenBank to avoid inadvertent sequence homologies. They were designed and synthesised by Sangon Biotechnology (Shanghai, China). Reactions were performed using SYBR Green PCR master mix (Applied Biosystems) in a BioRadiCycleriQ Detection System. As an internal control, 
TABLE 2: Serum uric acid levels in each group at different time points $(\mu \mathrm{mol} / \mathrm{L}$, mean $\pm \mathrm{SD})$.

\begin{tabular}{lccccc}
\hline Group & $N$ & Week 0 & Week 3 & Week 5 & Week 7 \\
\hline Control & 7 & $89.56 \pm 14.18$ & $104.35 \pm 12.49$ & $128.56 \pm 23.96^{*}$ & $121.38 \pm 12.66^{*}$ \\
OA & 7 & $91.69 \pm 19.31$ & $226.45 \pm 46.26$ & $224.8 \pm 90.59$ & $222.3 \pm 102.41$ \\
OA + Allopurinol & 7 & $81.47 \pm 26.17$ & $119.93 \pm 32.24$ & $179.01 \pm 58.23^{*}$ & $137.57 \pm 42.71^{* \#}$ \\
OA + SZF & 7 & $72.95 \pm 25.57$ & $183.96 \pm 82.93$ & $210.65 \pm 54.39$ & $167.26 \pm 82.94^{*}$ \\
\hline
\end{tabular}

${ }^{*} P<0.05$ versus the $\mathrm{OA}$ group. ${ }^{\#} P>0.05$ versus the $\mathrm{OA}+$ Allopurinol group.

TABLE 3: Total urine uric acid excretion in each group (mean \pm SD).

\begin{tabular}{lcccc}
\hline Group & $N$ & Urine volume $(\mathrm{L} / 24 \mathrm{~h})$ & Uua $(\mu \mathrm{mol} / \mathrm{L})$ & Uua $(\mu \mathrm{moL} / 24 \mathrm{~h})$ \\
\hline Control & 7 & $0.036 \pm 0.01$ & $1969.34 \pm 808.21$ & $64.00 \pm 20.78^{*}$ \\
OA & 7 & $0.034 \pm 0.01$ & $4271.16 \pm 2429.5$ & $124.39 \pm 29.39$ \\
OA + Allopurinol & 7 & $0.051 \pm 0.00$ & $4102.0 \pm 1576.09$ & $211.91 \pm 88.69^{* \#}$ \\
OA + SZF & 7 & $0.045 \pm 0.02$ & $5254.3 \pm 3916.99$ & $186.51 \pm 24.72^{*}$ \\
\hline
\end{tabular}

${ }^{*} P<0.05$ versus the OA group. ${ }^{\#} P>0.05$ versus the $\mathrm{OA}+$ Allopurinol group.

rGAPDH levels were quantified in parallel with the target genes. Normalisation and fold changes for each gene were calculated using the 2-DeltaDelta $\mathrm{C}(\mathrm{T})$ method.

2.10. Western Blot. Approximately $100 \mathrm{mg}$ of frozen rat kidney tissues was homogenised in $1 \mathrm{ml}$ of RIPA buffer and then centrifuged at $10,000 \mathrm{~g}$ for $20 \mathrm{~min}$. The protein concentrations of the supernatants were measured according to the Bradford method. Total proteins were denaturalized in boiling water for $5 \mathrm{~min}$. Equal amounts of total protein were separated onto $6 \%-12 \%$ SDS-PAGE and electrophoretically transferred to a polyvinylidenedifluoride (PVDF) membrane (Millipore, Shanghai, China) which was preactivated with methanol in the transferring buffer. Membranes were blocked with 5\% skim milk for $2 \mathrm{~h}$ and incubated overnight with specific primary antibodies at $4^{\circ} \mathrm{C}$. Immunoreactive bands were detected using HRP-conjugated goat anti-rabbit IgG as the secondary antibody (1:5000) (Jackson, Shanghai, China). Immunoreactive bands were visualised using a phototopehorseradish peroxidase Western blotting detection system (Cell Signaling Technologies, Beverly, MA) and quantified through densitometry with Molecular Analyst (Bio-Rad Laboratories, Hercules, CA). Primary antibodies included rabbit polyclonal antibodies against rTXNIP, rNLRP3, rASC, rCaspase-1, rpro-IL-1 $\beta$, rIL-1 $\beta$, and pro-IL-18, and rIL-18 (all $1: 1000)$.

2.11. Measurement of Serum IL-1 $\beta$ and IL-18 Levels. IL- $1 \beta$ and IL-18 levels in serum were determined by the ELISA kits following the manufacturer's instruction.

2.12. Statistical Analysis. Data are expressed as the mean \pm standard error of the mean or standard deviation (SD). Statistical analysis was performed by a one-way analysis of variance (ANOVA) followed by the Student-Newman-Keuls test. Differences were considered significant at $P<0.05$. The figures were obtained using SPSS version 18.0.

\section{Results}

3.1. SZF Reduces Oxonic Acid Potassium-Induced Serum Uric Acid in Hyperuricemic Rats, Ameliorates Renal Inflammation and Injury, and Inhibits Collagen Proliferation. In clinical settings, serum uric acid in hyperuricemic patients is approximately 1.5 to 2 times higher than that in healthy individuals. Hyperuricemia typically develops into a chronic, progressive condition. Therefore, we used an animal model of mild hyperuricemia resembling clinical characteristics by mixing $2 \%$ oxonic acid potassium salt (OA) into the diet of male Sprague-Dawley rats to induce nonurate deposition [21]. The rats in the treatment groups were generally in good condition, and none of them died during the experimental process. The changes in animal weight are illustrated in Figure 2(a). Observation of the control, OA model, and treatment groups indicated significant differences $(P<0.05)$. The Sua levels of the lab rats at Weeks $0,3,5$, and 7 as well as their $24 \mathrm{~h}$ urinary UA excretion measurements are illustrated in Tables 2 and 3 and Figure 2(b). At the end of Week 7, the Sua levels of the rats in the model group were approximately 1.8 times higher than those in the control group (OA group: $223.3 \pm$ $102.41 \mu \mathrm{mol} / \mathrm{L}$; controls: $121.38 \pm 12.66 \mu \mathrm{mol} / \mathrm{L})$, which was consistent with the clinical characteristics of mild elevation of serum uric acid. A comparison between the two treatment groups showed no significant statistical differences $(P>$ $0.05)$, suggesting that SZF and allopurinol achieved similar effects in reducing Sua. The total $24 \mathrm{~h}$ urinary UA excretion volume of the model group was significantly higher than that of the control group $(P<0.05)$, and those of the SZF and allopurinol groups were significantly higher than that of the OA group $(P<0.05)$. These observations suggest that the SZF effectively reduced Sua by promoting UA excretion.

Allopurinol is a widely recognised xanthine oxidase (XOD) inhibitor. It suppresses XOD activity and disrupts UA production, thereby reducing the effects of UA. In this study, the urinary UA excretion volume of the allopurinol group far exceeded that of the control and OA groups, verifying the 


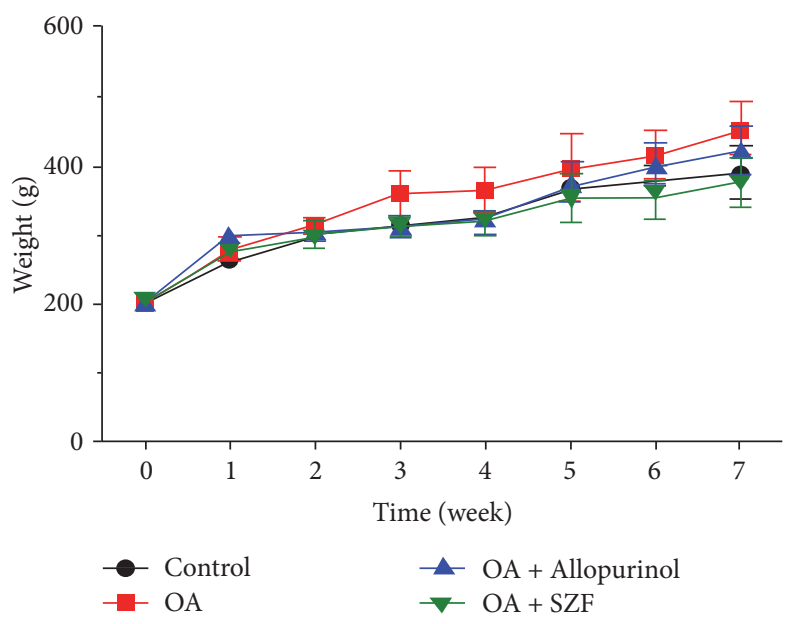

(a)
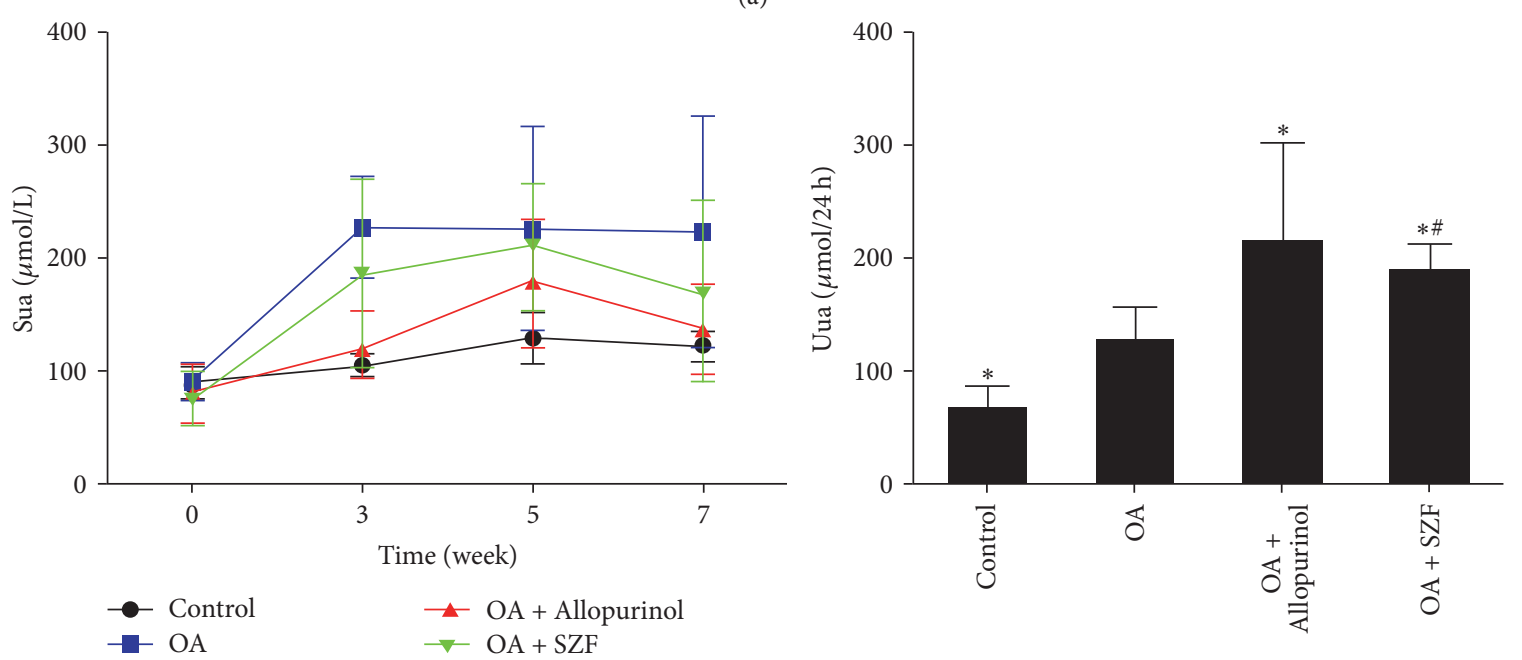

(b)
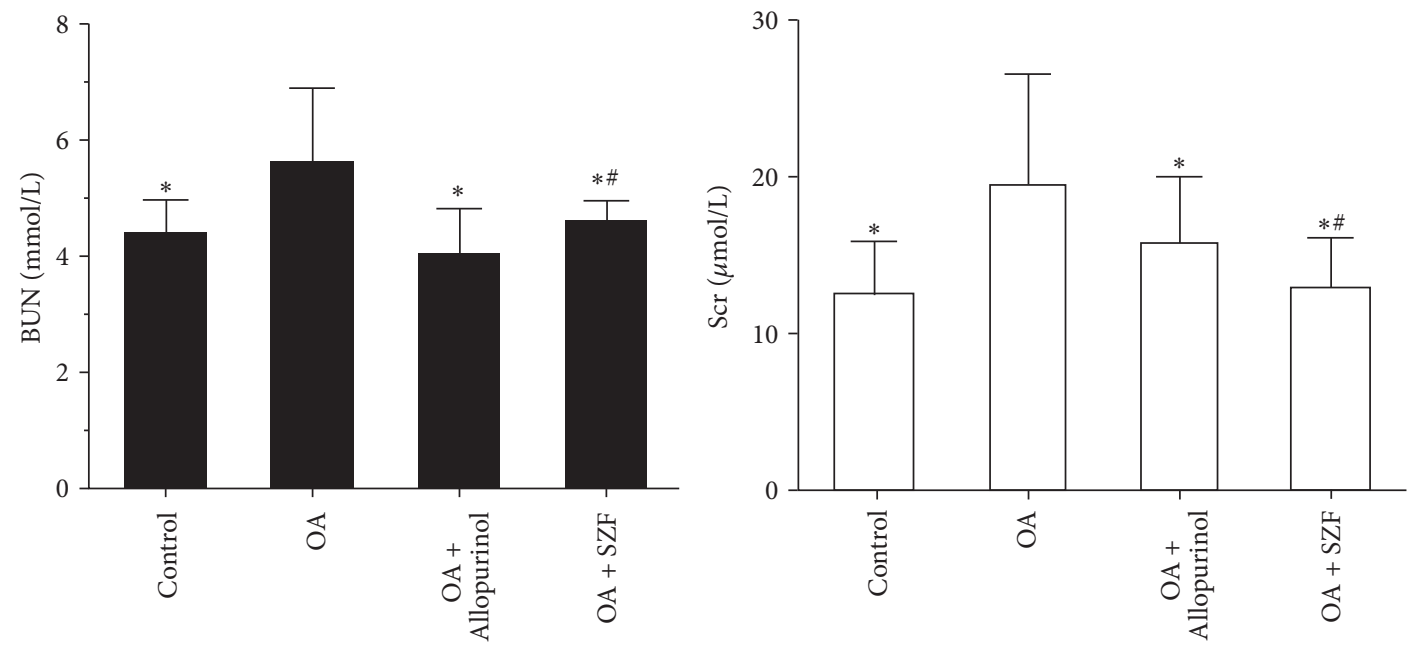

(c)

Figure 2: SZF improved the kidney function of rats with OA-induced hyperuricemia. (a) Changes in animal weight over the 7 weeks of animal modelling and treatment; OA: oxonic acid potassium salt; (b) changes in Sua in the tail vein over the 7 weeks of animal modelling and treatment and the $24 \mathrm{~h}$ urinary UA excretion conditions at the end of the 7 weeks; UA: uric acid; Sua: serum uric acid; Uua: $24 \mathrm{~h}$ urinary uric acid excretion; (c) BUN and Scr levels in the abdominal aorta of the various groups at the end of the 7 weeks of animal modelling and treatment. BUN: blood urea nitrogen; Scr: serum creatinine. Control: the rats received standard diet and no intervention; OA: the rats received $2 \%$ OA diet to produce hyperuricemic model; OA + Allopurinol: the rats received $2 \%$ OA diet and drinking water dissolved in allopurinol; OA + SZF: the rats received 2\% OA diet and Traditional Chinese Medicine formula of Shizhifang intragastrically. Data are expressed as the mean $\pm \mathrm{SD}(n=7) .{ }^{*} \mathrm{P}<0.05$ versus the OA group. ${ }^{\#} P>0.05$ versus the $\mathrm{OA}+$ Allopurinol group. 

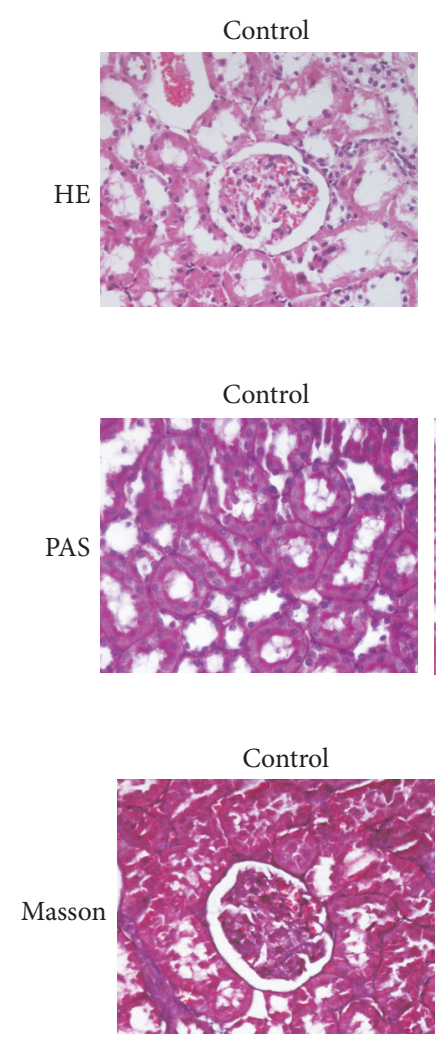

$\mathrm{OA}$

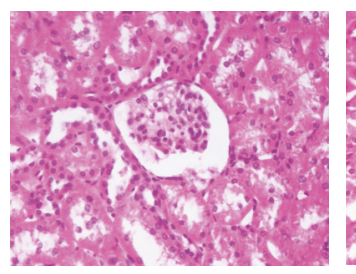

(a)

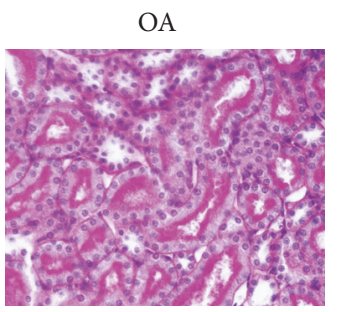

$\mathrm{OA}+$ Allopurinol

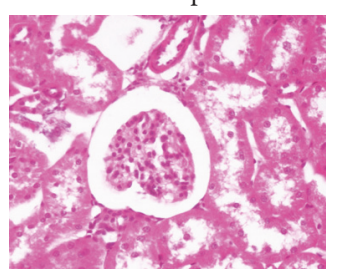

$\mathrm{OA}+$ Allopurinol

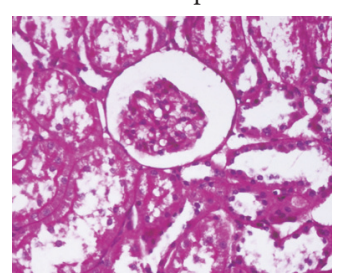

$\mathrm{OA}+\mathrm{SZF}$

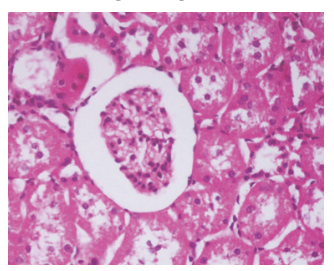

$\mathrm{OA}+\mathrm{SZF}$

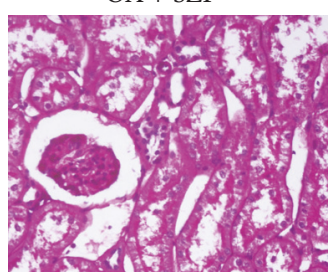

(b)
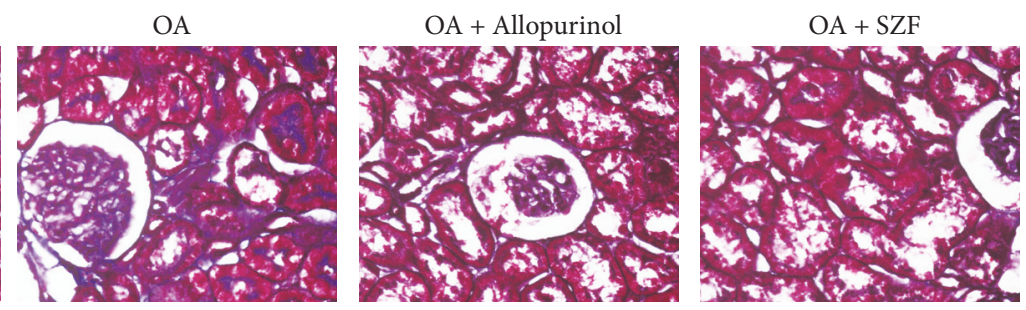

(c)

FIGURE 3: SZF improved renal pathological morphology and reduced cell damage in the hyperuricemic rats. (a) H\&E staining results of the renal tissue (magnification 400x); (b) PAS staining results of the renal tissue (magnification 400x); (c) Masson's staining results of the renal tissue (magnification 400x).

TABLE 4: Blood urea nitrogen and serum creatinine of rats in each group (mean $\pm S D)$.

\begin{tabular}{lccc}
\hline Group & $N$ & BUN $(\mathrm{mmol} / \mathrm{L})$ & Scr $(\mu \mathrm{mol} / \mathrm{L})$ \\
\hline Control & 7 & $4.40 \pm 0.56^{*}$ & $12.45 \pm 3.43^{*}$ \\
OA & 7 & $5.58 \pm 1.30$ & $19.54 \pm 6.94$ \\
OA + Allopurinol & 7 & $3.99 \pm 0.82^{*}$ & $12.93 \pm 3.15^{*}$ \\
OA + SZF & 7 & $4.57 \pm 0.40^{* \#}$ & $15.79 \pm 4.17^{\#}$ \\
\hline
\end{tabular}

${ }^{*} P<0.05$ versus the $\mathrm{OA}$ group. ${ }^{\#} P>0.05$ versus the $\mathrm{OA}+$ Allopurinol group.

effects of allopurinol on UA excretion. These observations are consistent with those reported in a previous study [22]. The serum creatinine and blood urea nitrogen levels of the various groups at the end of Week 7 are shown in Figure 2(c) and Table 4. Compared with the controls, the BUN and Scr levels of OA group were significantly higher $(P<0.05)$, verifying that elevated UA damaged kidney function. However, the BUN and Scr levels of the lab rats receiving the SZF and allopurinol treatments decreased.

Haematoxylin and eosin (H\&E) staining results showed that the kidney tissue structures of the controls were relatively normal (Figure 3(a)). Local swelling in the renal proximal tubular epithelial cells and significant increases in vacuolar degeneration and inflammatory cell infiltration were observed in the model group. In addition, a small portion of tubular epithelial cells exhibited an increase in epithelial cell nuclei shedding due to vacuolar degeneration. No differences were observed in the glomeruli. For the OA + SZF and OA + Allopurinol groups, there was a similar lack of changes in the glomeruli. These groups exhibited reduced swelling in the renal proximal tubular epithelial cells, vacuolar degeneration, and inflammatory cell infiltration. According to the Periodic Acid-Schiff (PAS) and Masson's trichrome staining results, the controls showed orderly renal tubule arrangements, uniform cell walls, normal brush-border microvilli formation on the inner surface of the tubular lumens, basement folds formed from basal cell invagination, and no renal interstitial fibrosis (Figures 3(b) and 3(c)). The OA group showed disorderly renal tubule arrangements, inflamed tubules and interstitial cell infiltration, narrow lumens, changes in the brush-border microvilli structures of the tubular walls, partial shedding, incoherent basal membranes, and fibrosis of 
the tubular lumens and interstitia. The OA + Allopurinol group showed orderly renal tubule arrangement, slight tubular inflammation and interstitial cell infiltration, expanded lumens, vacuolar degeneration, changes in the brush-border microvilli structures of the tubular walls, incoherent basal membranes, and slight fibrosis in the tubular lumens and interstitia. The OA + SZF group showed orderly renal tubule arrangements, uniform and consistent cell walls, rough luminal surfaces, no evident changes in microvilli structures, basal cell invagination forming basement folds, and no evident fibrosis in the tubular lumens and interstitia.

3.2. SZF Inhibits OS in Hyperuricemic Lab Rats by Suppressing Mitochondrial ROS. Physiologically, ROS serve as secondary messengers for cell signals and protein modifications. They control cell growth and proliferation and regulate the transcription and activity of different molecules. Excess release of ROS may trigger renal tissue and body OS, change redox states, permanently damage large molecules (e.g., DNA, RNA, proteins, and lipids), and interfere with crucial signalling pathways for redox reactions [23]. In this study, we tested the ROS expression in the serum and renal tissue of rats with OA-induced hyperuricemia (Figure 4(a)). As anticipated, serum collected from the rats in the OA group showed elevated ROS levels $(P<0.05$; statistically significant) and renal tissue $(P>0.05$; statistically nonsignificant). By comparison, the treatment groups showed similar effects in suppressing ROS release. To identify the sources of renal ROS in hyperuricemic rats with UA-induced renal inflammation, we obtained mitochondria from renal tissue, performed mitochondria isolation, and tested the ROS levels in the mitochondria (Figure 4(b)). The results verified that mitochondrial ROS serves a key function in the renal tissue and in vivo OS. These observations are consistent with the findings of Ives et al. [24], who contended that mitochondrial ROS originating from XOD is the main source of ROS for kidney inflammasome activation. Furthermore, we tested the OS level of the hyperuricemic rats in their serum and renal tissue (Figures 4(c) and 4(d)) and found a significant imbalance between the superoxide dismutase (SOD), catalase (CAT), and glutathione peroxidase (GSH-PX) of the antioxidant systems and the malondialdehyde (MDA) of the peroxidation systems in the rats' bodies and specifically kidneys. Subsequently, SZF and allopurinol intervention restored the dynamic balance of these compounds to a certain extent. To elucidate the influence of OS on renal tissue inflammation and cell damage, we applied immunohistochemistry to test the contents of the tyrosine-specific nitration products (3NT; Figure 5(a)) and lipid peroxide (4-HNE; Figure 5(b)) in various groups. The results indicated that 3-NT and 4HNE were primarily expressed in the cytoplasm of tubular epithelial cells. The OA group exhibited elevated 3-NT and 4-HNE expression in the renal interstitia, suggesting that OS damages the kidneys and that SZF can reduce OS-induced renal inflammation and tubular injury. These results also suggest that high UA levels can prompt mitochondria to produce excess ROS, leading to an imbalance between renal and in vivo oxidation and antioxidation systems and thereby causing OS and an increased probability of renal damage. SZF can repair OS-induced injury. To elucidate the interaction between UA-stimulated ROS and renal inflammation, we examined the effects of UA on the pathways concerning renal anti-inflammation, NLRP3 inflammasome activation, and SZF intervention.

3.3. SZF Inhibits Activation of the NLRP3-ASC-Caspase-1 Axis by Suppressing TXNIP. UA activates the inflammasome molecular complex NLRP3 in the cells through the ROSTXNIP pathway, leading to the maturation and release of inflammatory factors IL- $1 \beta$ and IL-18. We performed realtime PCR and Western blotting to test the TXNIP mRNA and protein expression in the renal tissue of the hyperuricemic rats. Unsurprisingly, TXNIP mRNA and protein expression in the renal tissue of the rats in OA group were significantly higher than those in the other groups $(P<0.05)$. SZF and allopurinol effectively suppressed TXNIP mRNA and protein expression. SZF was particularly exceptional in suppressing TXNIP (Figure 6(a)). We then examined the influences of SZF on the activation index of the downstream NLRP3-ASCcaspase- 1 axis in the TXNIP. First, we tested the mRNA and proteins in the NLRP3-ASC-caspase-1 axis (Figures 6(b) $-6(d)$ ) and found elevated mRNA and protein expression in the hyperuricemic rats $(P<0.05)$. SZF effectively suppressed the activation of the NLRP3-ASC-caspase-1 axis, indicating that the suppression effect was due to suppression of the ROS-TXNIP pathway (OS index). Second, we tested the mRNA and protein expression of the downstream inflammatory factors Pro-IL-18, IL-18, Pro-IL-1 $\beta$, and IL-1 $\beta$ in the NLRP3-ASC-caspase-1 axis (Figures $7(\mathrm{a})$ and $7(\mathrm{~b})$ ). Moreover, we detected the IL- $1 \beta$ and IL-18 levels in serum by the ELISA kits (Figure $7(\mathrm{c})$ ). Common results showed that SZF effectively reduced the inflammatory cytokines levels of IL-1 $\beta$ and IL-18 in serum and kidney tissues of hyperuricemic rats $(P<0.05)$. Immunohistochemical staining was adopted for a localization analysis (Figure 8 ). The results showed that NLRP3, ASC, caspase-1, IL-18, and IL-1 $\beta$ were all present in the tubular epithelial cytoplasm and were rarely expressed in the glomeruli. Examination of the real-time PCR, Western blotting, and immunohistochemistry results verified that SZF could effectively suppress the maturation and release of IL18 and IL-1 $\beta$, thus inhibiting renal inflammation. According to the results, UA stimulates excess ROS production and activates $O S$ in the kidneys, which affects TXNIP activity and prompts TXNIP to bond with NLRP3, further triggering the assembly of the NLRP3 inflammasome. This process continues to activate the NLRP3-ASC-caspase-1 axis to release inflammatory factors and trigger renal inflammatory responses. SZF suppresses the activation of the NLRP3-ASCcaspase- 1 axis by inhibiting TXNIP, thus ameliorating renal inflammation.

\section{Discussion}

An increasing amount of evidence suggests that UA-induced inflammatory responses are the central mechanisms of tubular injury in hyperuricemic rodents [25] and that the activation of NLRP3 and its mediating effects on inflammatory responses play key roles in various renal diseases [26]. 

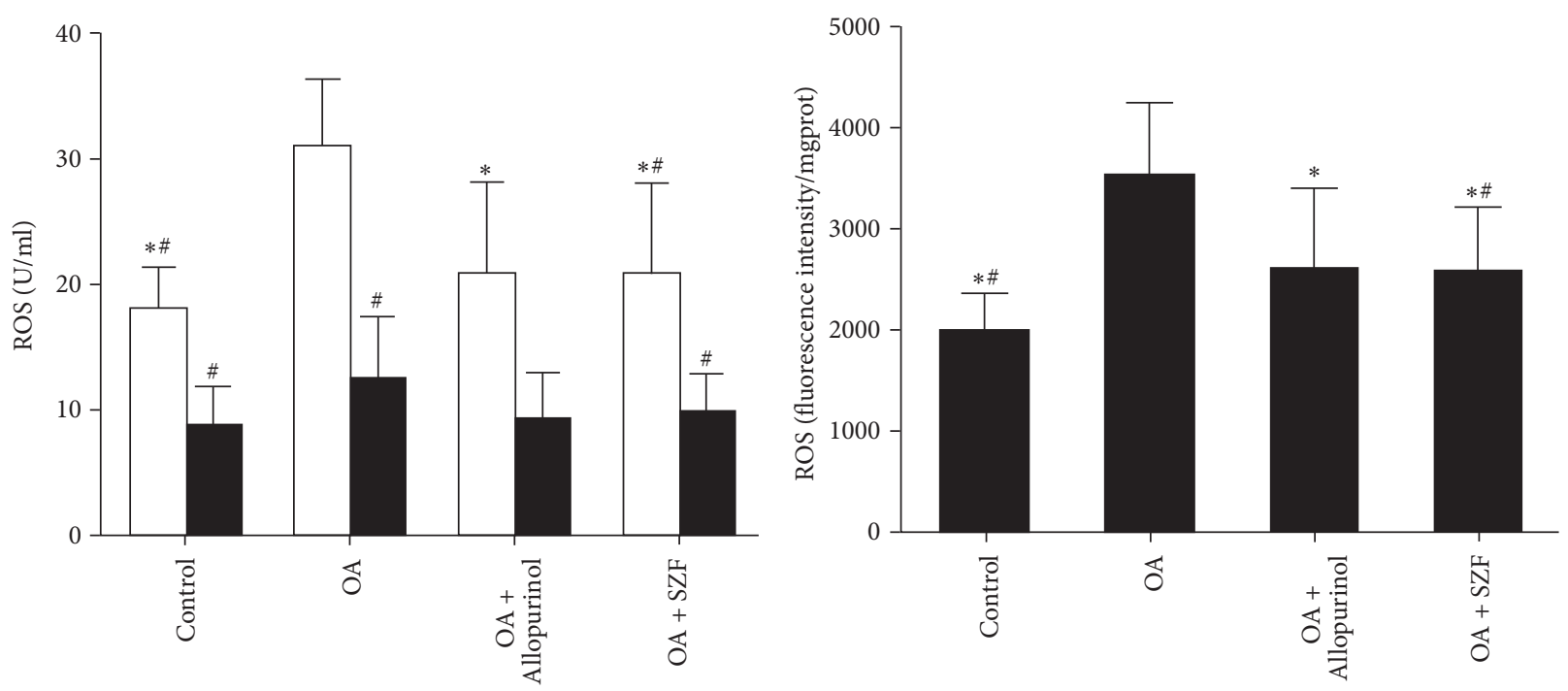

$\square$ ROS of serum

ROS of kidney tissues

(a)

(b)
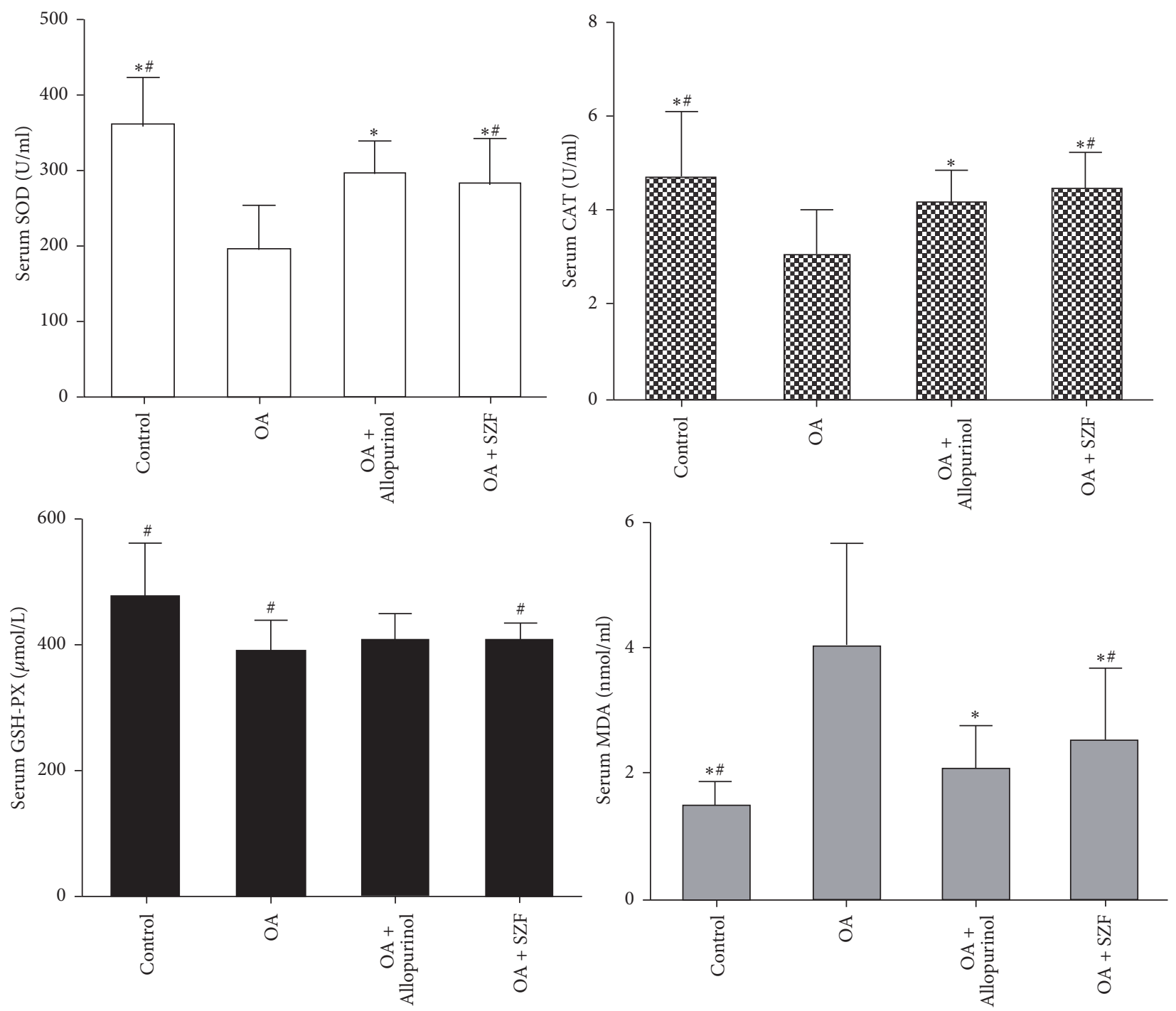

(c)

Figure 4: Continued. 

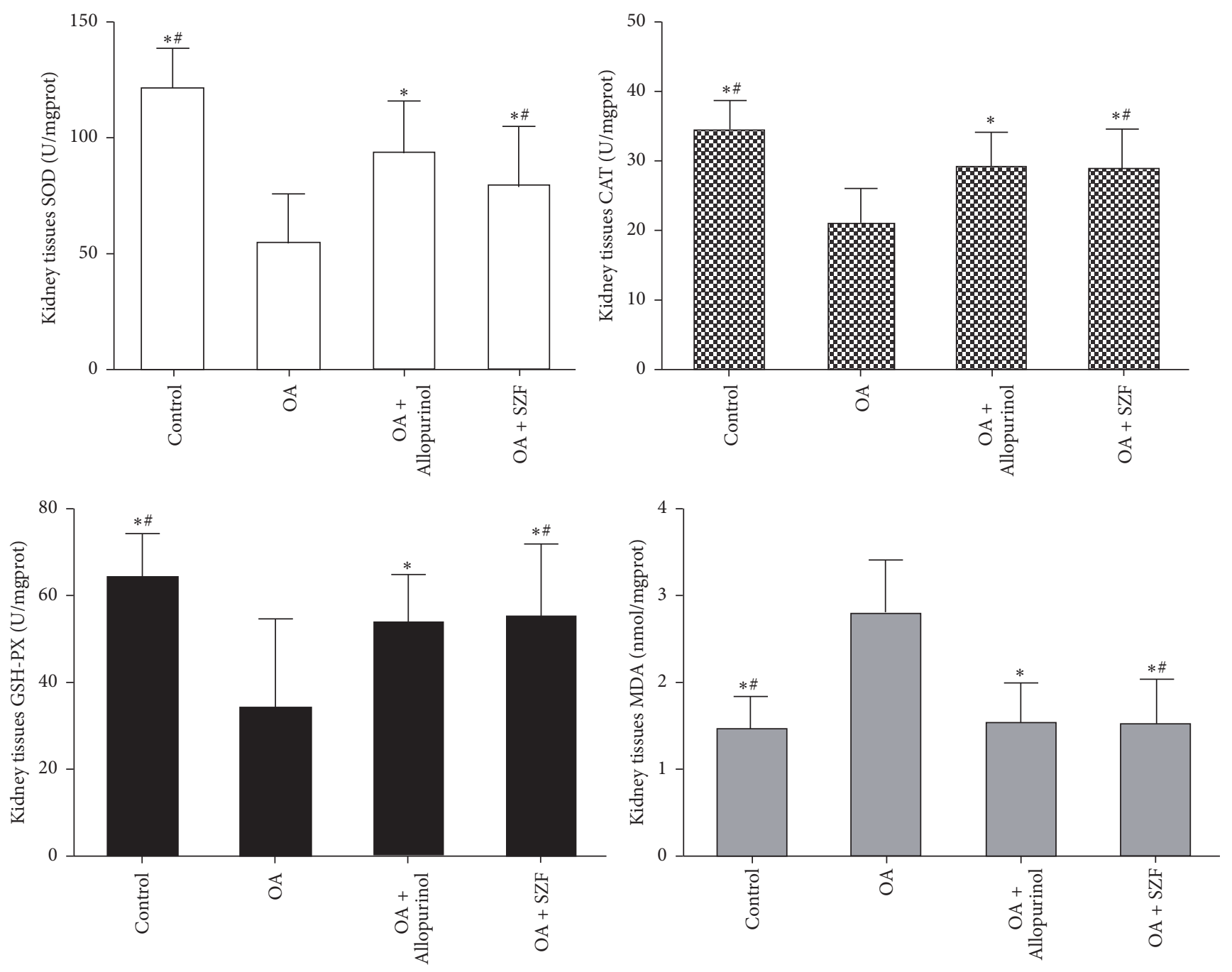

(d)

FIGURE 4: SZF inhibited the OS of hyperuricemic rats by suppressing mitochondrial ROS. (a) ROS levels in the serum and renal cortex tissue; ROS: reactive oxygen species; OS: oxidative stress; (b) relative fluorescence intensity of the mitochondrial ROS after the mitochondria were isolated from the tissue cells of the renal cortex; (c) SOD, CAT, GSH-PX, and MDA levels in the serum of the various groups; SOD: superoxide dismutase; CAT: catalase; GSH-PX: glutathione peroxidase; MDA: malondialdehyde; (d) SOD, CAT, GSH-PX, and MDA levels in the renal tissue of the various groups. Data are expressed as the mean $\pm \mathrm{SD}(n=7) .{ }^{*} P<0.05$ versus the OA group. ${ }^{*} P>0.05$ versus the OA + Allopurinol group.

Cristóbal-García et al. [27] found that OA-induced longterm hyperuricemia promoted OS in the renal cortex, damaged mitochondria, and reduced ATP levels. Park et al. [28] proposed the dynamics of mitochondrial damage, explaining that defective mitochondrial fission augments NLRP3 inflammasome activation and triggers abnormal inflammatory responses, confirming that NLRP3 activation and NLRP3-mediated inflammatory responses significantly influence inflammation in renal interstitia. The findings of numerous previous studies have verified that mitochondrial ROS activates the NLRP3 inflammasome through TXNIP, triggering a rapid renal inflammation response. As a sensitive signal complex regulator in cell redox, Trx/TXNIP has become a key point of connection between redox regulation and disease [29]. During cell quiescence, TXNIP and Trx1 combine and remain deactivated. Trx-1 controls the ROS levels in cells and suppresses OS. When high levels of ROS are present in cells in a state of OS, TXNIP separates from Trx-1 and bonds with NLRP3 to activate the inflammasome and regulate inflammation signals $[30,31]$. In this study, we observed high expression of mitochondrial ROS and TXNIP in the renal tissue under elevated UA conditions, which triggered NLRP3 overexpression and renal inflammation. However, ROS was suppressed by the allopurinol and SZF interventions, in turn suppressing OS and maintaining the TXNIP-Trx bond. This bond suppressed TXNIP activity and 


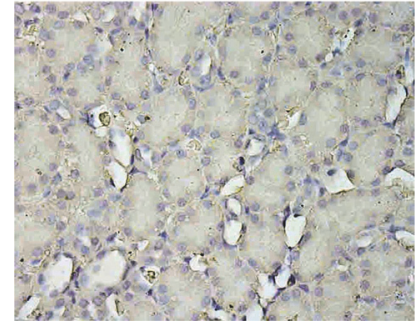

Control

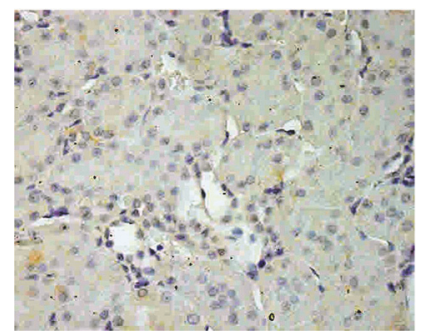

$\mathrm{OA}+$ Allopurinol

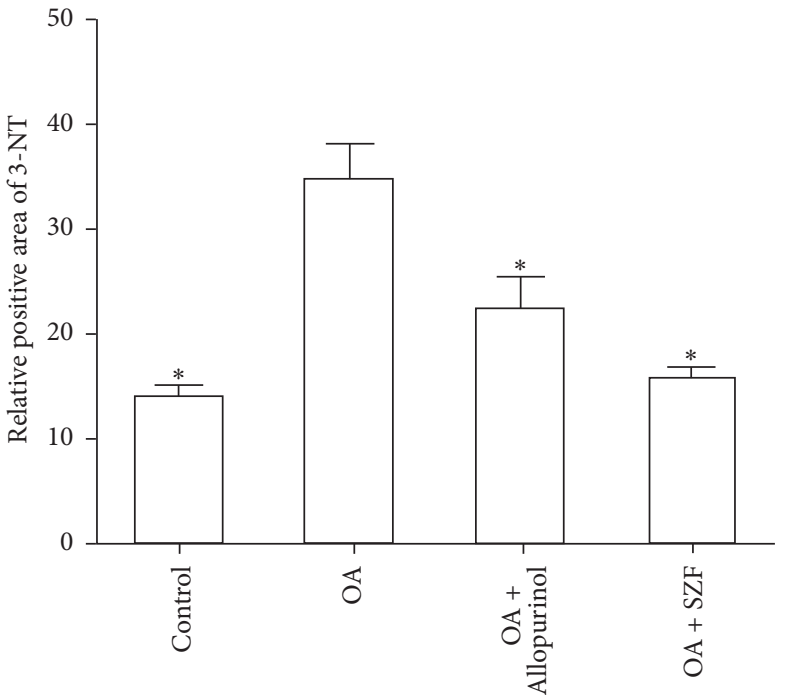

(a)

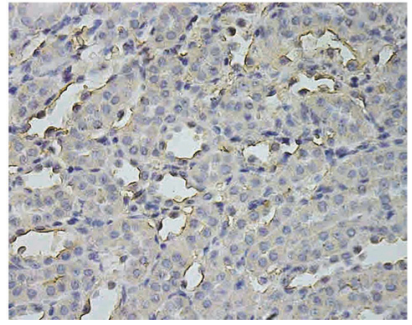

$\mathrm{OA}$

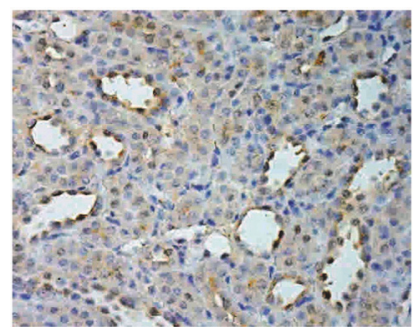

$\mathrm{OA}+\mathrm{SZF}$

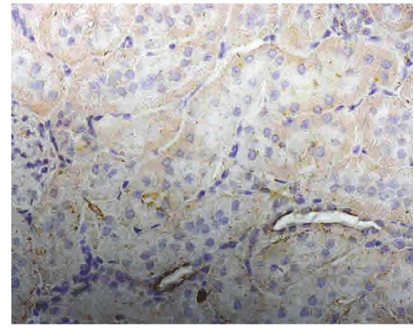

Control

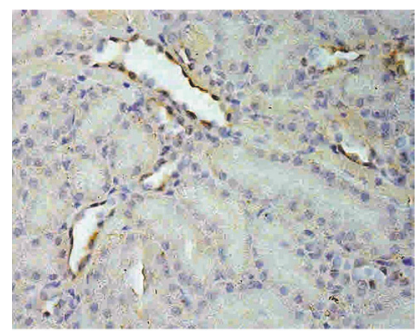

$\mathrm{OA}+$ Allopurinol

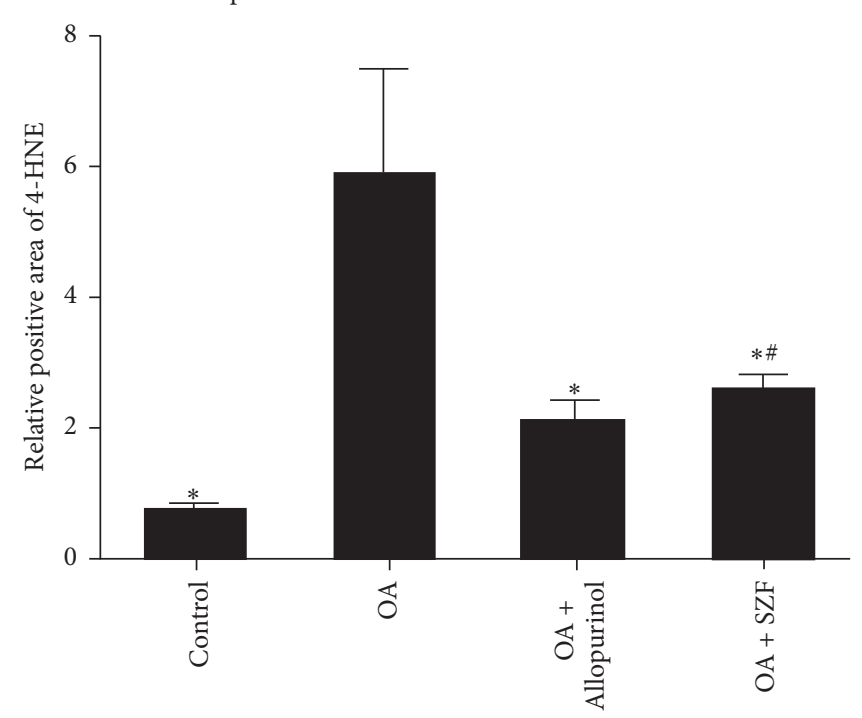

(b)

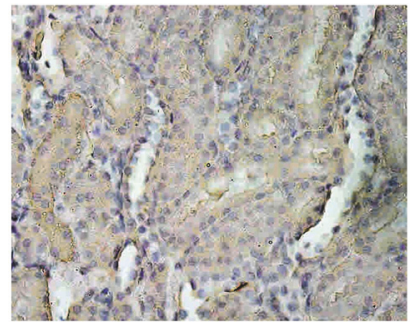

$\mathrm{OA}$

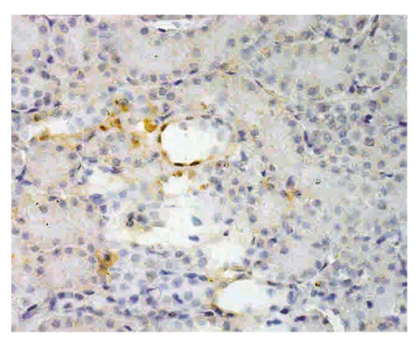

$\mathrm{OA}+\mathrm{SZF}$

FIGURE 5: SZF affected the expression of the OS products 3-NT and 4-HNE in renal tissue with high UA. (a) 3-NT immunohistochemical staining (magnification 200x) and positive area results in the renal tissue; 3-NT: 3-nitrotyrosine; (b) 4-HNE immunohistochemical staining (magnification 400x) and positive area results in the renal tissue; 4-HNE: 4-hydroxy aldehyde. Data are expressed as the mean \pm SD $(n=7)$. ${ }^{*} P<0.05$ versus the $\mathrm{OA}$ group. ${ }^{*} \mathrm{P}>0.05$ versus the $\mathrm{OA}+$ Allopurinol group.

prevented it from bonding with NLRP3, thereby preventing the activation of the inflammasome axis and ameliorating renal inflammation and injury.

UA promotes activation of downstream caspase-1 in the NLRP3 inflammasome, creating a key platform for the activation and release of proinflammatory factors. The formation of myoblast caused by inflammatory cell aggregation and infiltration and the activation of inflammatory and proinflammatory factors are essential contributors to renal fibrosis, under which CKD progresses to ESRD. Immune inflammation triggers injury repair responses, leading to fibrosis and scarring and causing substantial function degradation. Ryu et al. [32] fed Sprague-Dawley rats standard feed containing 2\% OA for 6 weeks and found that UA promoted the epithelial-mesenchymal transition of tubular epithelial cells. Hyperuricemia-induced EMT occurred ahead of renal interstitial fibrosis. The reduction in E-cadherin expression and increase in $\alpha$-SMA expression indicated UA-induced phenotype transformation in the tubular epithelial cells. The activation of Snail and Slug transcription factors reduced Ecadherin synthesis and increased degradation of E-cadherin by ubiquitin, which promoted the phenotype transformation in the tubular epithelial cells. Mazzali et al. [21] analysed lab rats with OA-induced hyperuricemia and verified that hyperuricemia triggered systematic hypertension and ischaemic renal damage. Collagen deposition, macrophage infiltration, 

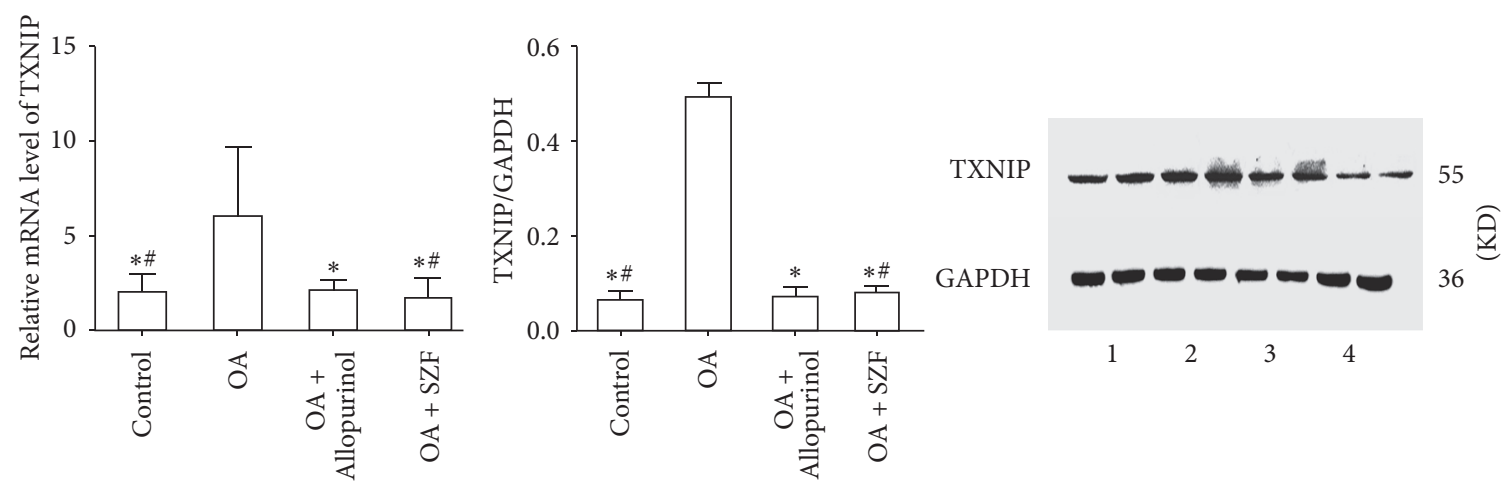

(a)
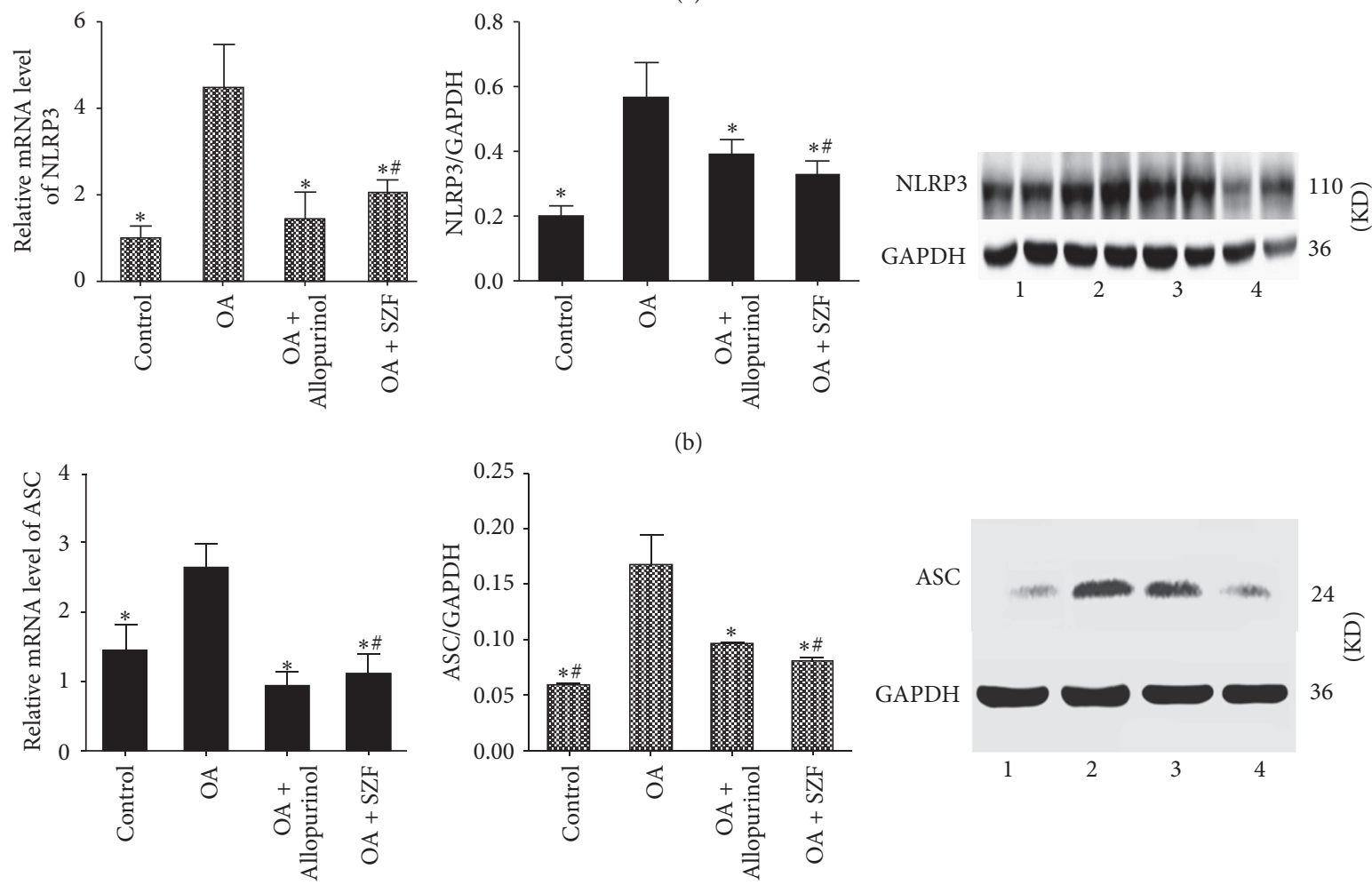

(b)
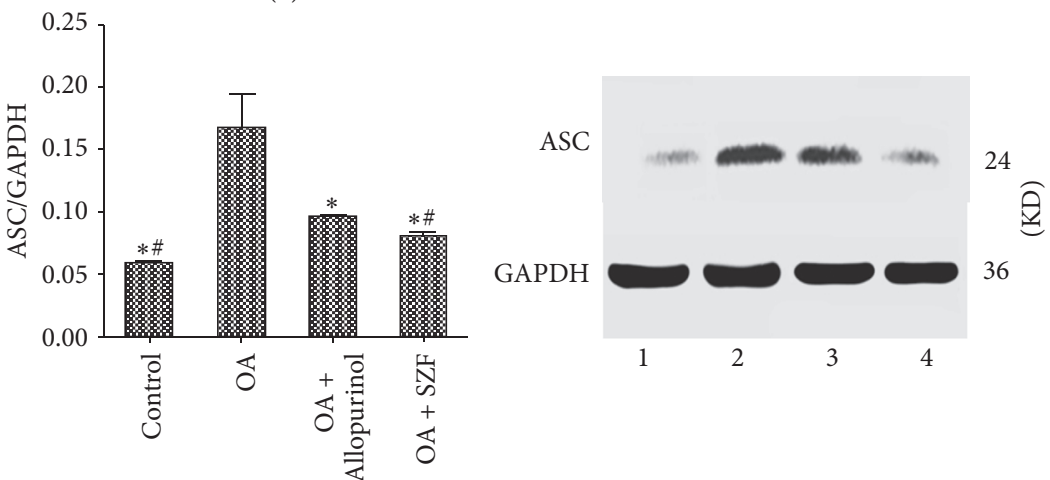

(c)
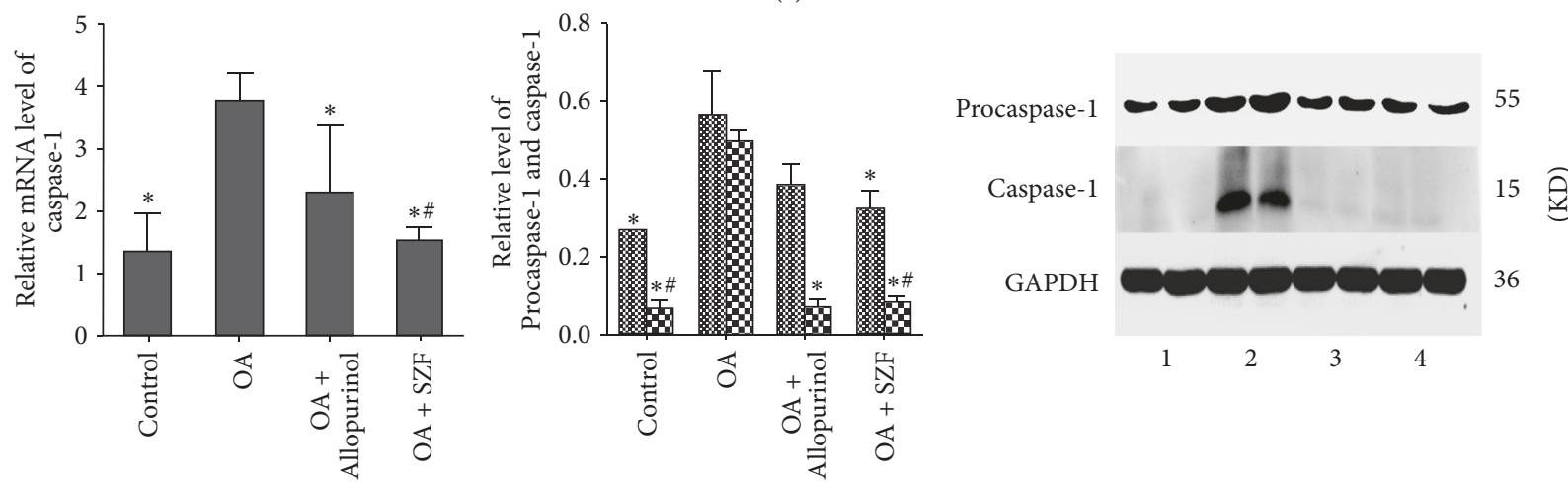

ॠ Procaspase-1

80 Caspase-1

(d)

FIGURE 6: SZF inhibited the activation of the NLRP3-ASC-caspase-1 axis by suppressing TXNIP. Gene and protein expression levels in the renal tissue: (a) TXNIP; (b) NLRP3; (c) ASC; (d) caspase-1 and Procaspase-1. Protein levels were determined by Western blotting, were quantified through densitometry, and are expressed as the optical density ratio to GAPDH. mRNA levels were determined through real-time PCR. The numbers in the Western blot figures represent groups: 1 means Control, 2 means OA, 3 means OA + Allopurinol, and 4 means OA + SZF. Data are expressed as the mean $\pm \mathrm{SD}(n=3) .{ }^{*} P<0.05$ versus the OA group. ${ }^{\#} P>0.05$ versus the OA + Allopurinol group. 

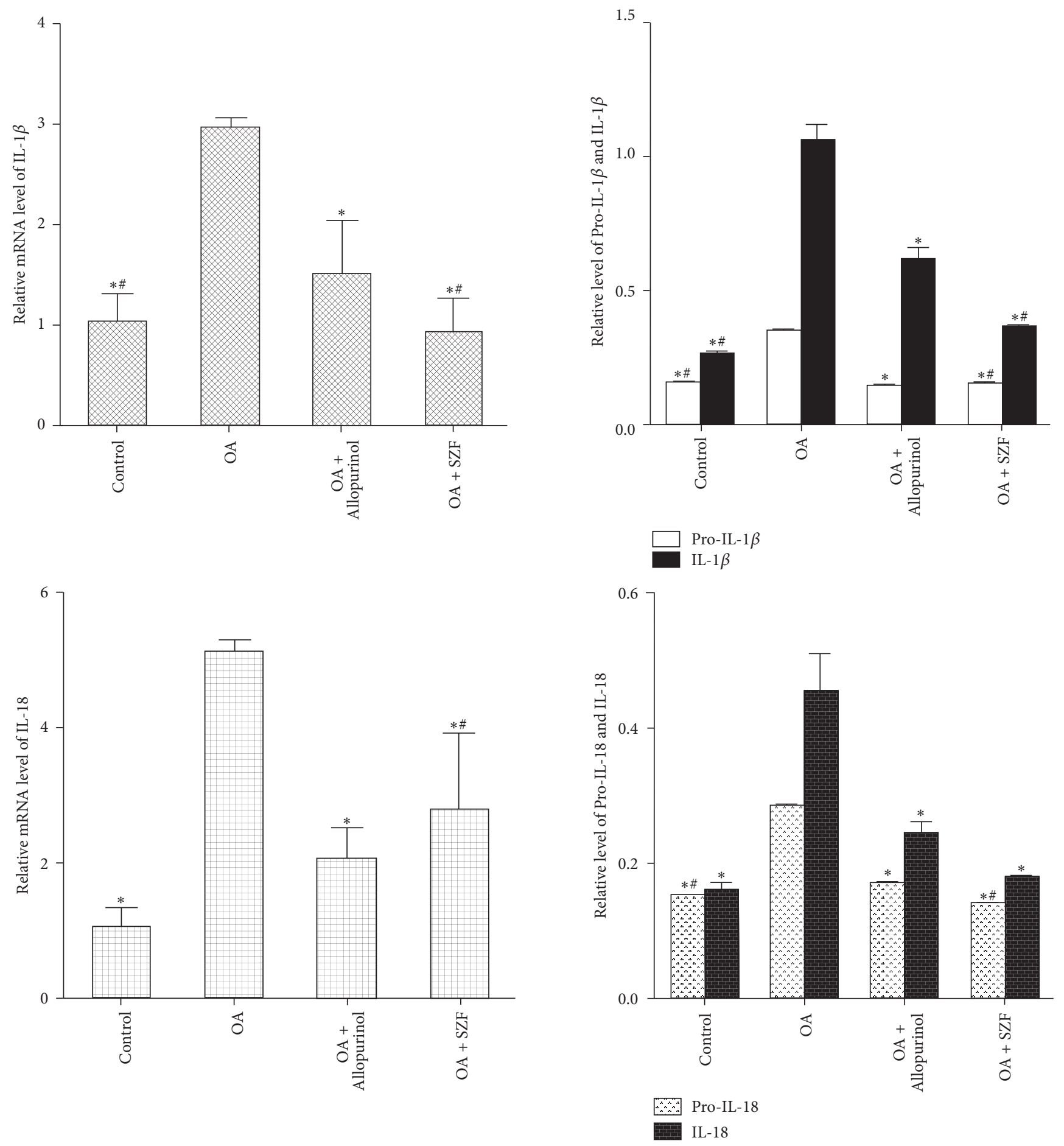

(a)

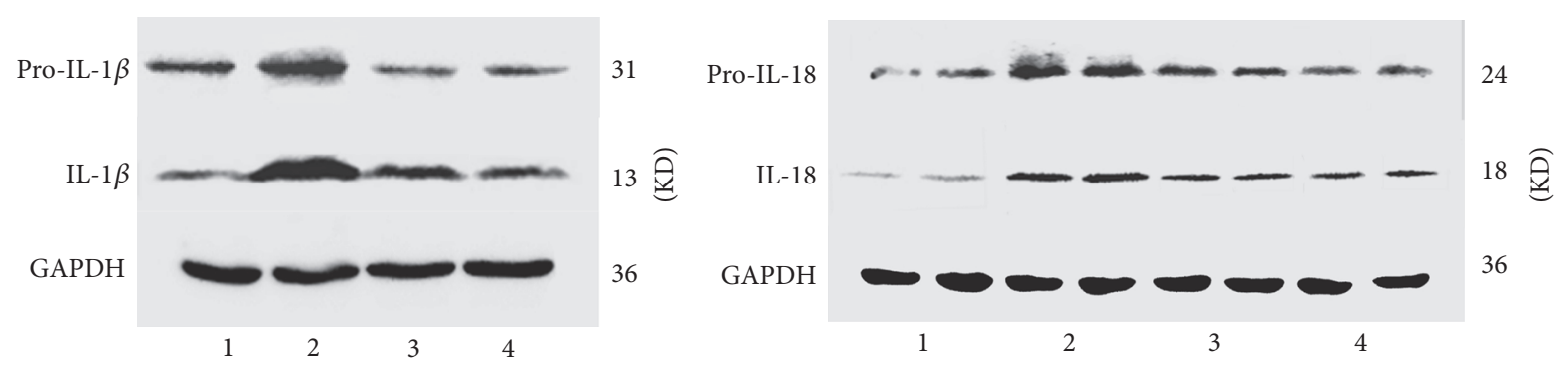

(b)

FIgURE 7: Continued. 

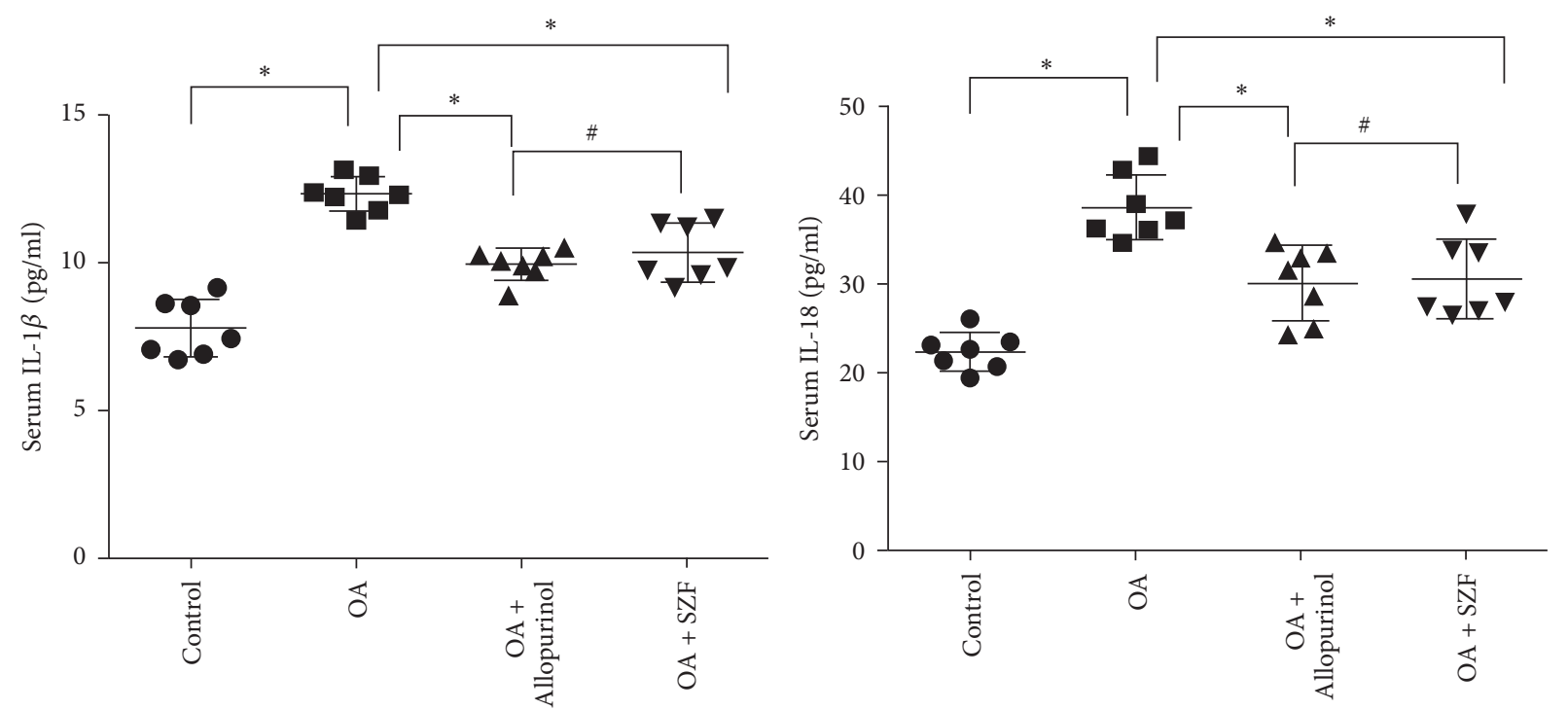

(c)

Figure 7: SZF reduced the levels of IL-1 $\beta$ and IL-18 in serum and kidney tissues of hyperuricemic rats. Gene and protein expression levels in the renal tissue: (a) The mRNA expression of IL-1 $\beta$ and IL-18 by Real-time PCR $(n=3)$; (b) The protein expression of IL-1 $\beta$ and IL-18 by Western blot $(n=3)$, The numbers in the Western blot figures represent groups, 1 means Control, 2 means OA, 3 means OA + Allopurinol, 4 means OA + SZF; (c) The serum inflammatory cytokines levels of IL- $1 \beta$ and IL-18 by ELISA $(n=7)$. Data are expressed as the mean \pm SD. ${ }^{*} P<0.05$ versus the OA group. ${ }^{*} P>0.05$ versus the $\mathrm{OA}+$ Allopurinol group.

and increased osteopontin expression were observed in the tubular and interstitial areas, suggesting that UA participates in renal inflammation and triggers tubular and interstitial fibrosis. Through PAS and Masson's trichrome staining, we verified that elevated UA levels could cause renal inflammation and interstitial fibrosis by activating the NLRP3 inflammasome. SZF suppressed this activation, thereby inhibiting renal interstitial inflammation and alleviating renal fibrosis.

NLRP3 controls the activation of caspase-1, which is a prerequisite for IL-1 $\beta$ and IL-18 maturation [33] and contributes to pyroptosis through canonical inflammasome pathway. Recently, Shi et al. [34] and Kayagaki et al. [35] have found that caspase-1 mediates the cleaving of gasdermin $\mathrm{D}$ (GSDMD) to form aminoterminal fragments that induce pyroptosis activity. The canonical inflammatory pathway caspase-1-GSDMD serves a decisive function in pyroptosis, triggering it when the inflammasome downstream molecule caspase-1 cleaves GSDMD [36]. Chen et al. [37] asserted that cadmium triggers the mediating effects of caspase- 1 on the pyroptosis and inflammation responses of vascular endothelial cells. The staining results of caspase-1 activation and the positive SYTOX green verified that cadmium activates the NLRP3 inflammasome in endothelial cells to trigger pyroptosis and produce ROS. Yang et al. [38] observed a significant increase in caspase- 1 and IL- $1 \beta$ proteins associated with pyroptosis in ischemia/reperfusion- (I/R) induced rats and discovered pyroptosis pore formation in NRK-52E cells and lactate dehydrogenase release during hypoxia and reoxygenation, verifying the occurrence of epithelial cell pyroptosis. Chung et al. [39] developed a unilateral ureteral obstruction (UUO) animal model of rats with kidney damage. Analysis outcomes showed tubular and interstitial inflammation infiltration in the obstructive side of the kidney in addition to several pathological changes such as fibrosis, autophagy, apoptosis, and pyroptosis. The UUO model exhibited increased expression of the caspase-1 and IL- $1 \beta$ proteins associated with pyroptosis in the kidneys. The application of catechin therapy using Nrf-2 agonist effectively protected mitochondrial function, improved renal blood flow, and significantly reduced the OS, inflammation, fibrosis, and three types of programmed cell death in kidneys with UUO.

Although we did not perform an in vitro analysis to observe the regulating effects of the SZF on cell pyroptosis, we investigated the changes in the expression levels of caspase1 , an activation protein for canonical pyroptosis pathways, and the subsequent effects of the maturity and release of inflammatory factors, thus deepening our understanding of pyroptosis in renal tissue. Our results indicated that the SZF effectively suppressed the activation of the NLRP3-ASCcaspase-1 axis, reducing the expression of caspase-1 proteins and preventing them from cleaving GSDMD. This process obstructs pyroptosis pathways, protecting the integrity of the cell membrane and ensuring normal cell function.

\section{Conclusion}

In summary, we verified that, through the suppression of mitochondrial ROS in the kidneys of hyperuricemic lab rats, SZF inhibits NLRP3 inflammasome activation, thereby alleviating tubular inflammation, cell pyroptosis, and renal interstitial fibrosis. We found that SZF demonstrated excellent 

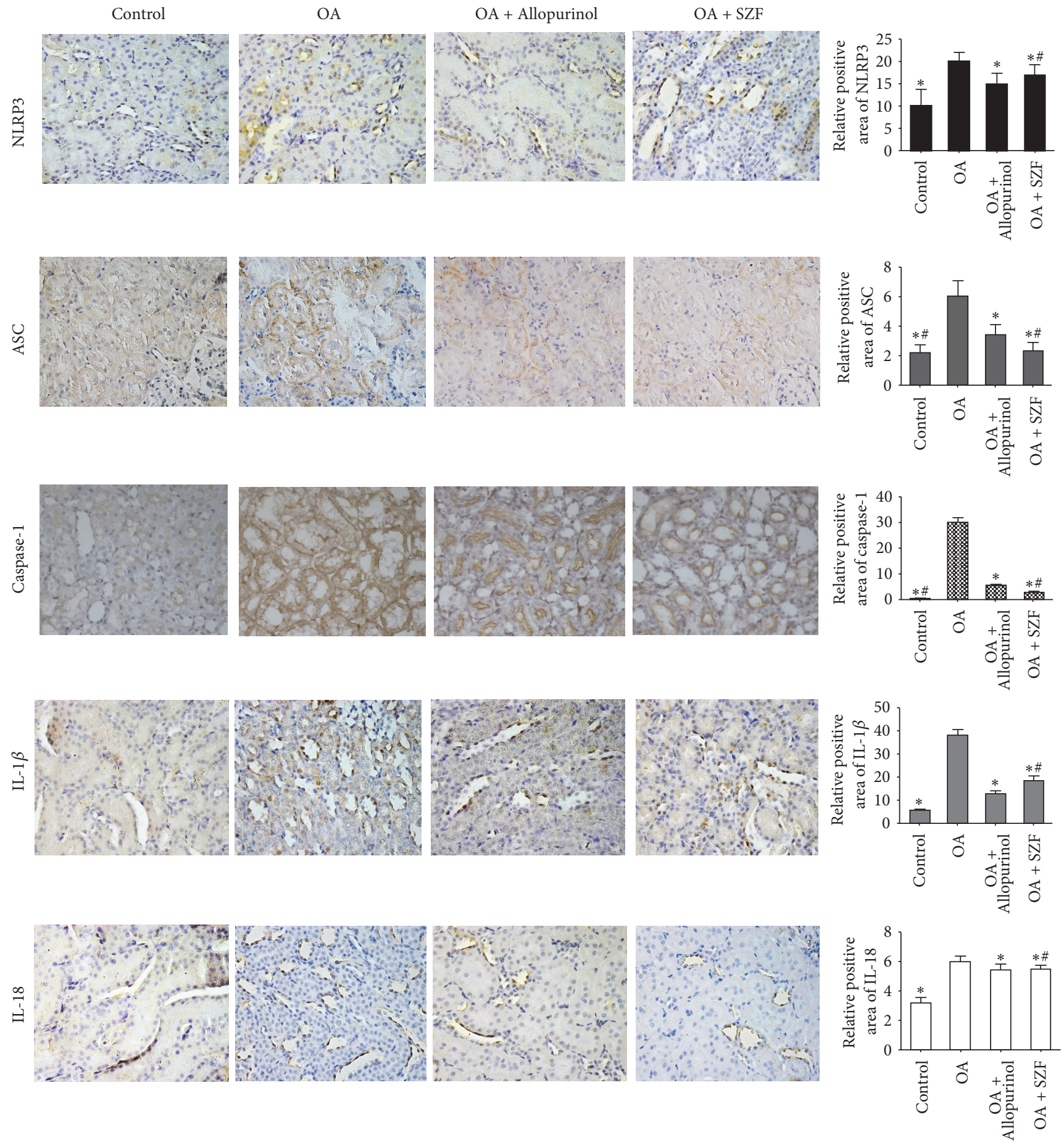

FIGURE 8: SZF affected the localization and expression of the NLRP3-ASC-caspase-1 axis proteins. NLRP3, ASC, caspase-1, IL-1 $\beta$, and IL-18 immunohistochemical staining (magnification 200x) and positive area results in the renal tissue. Data are expressed as the mean \pm SD $(n=7)$. ${ }^{*} P<0.05$ versus the OA group. ${ }^{\#} P>0.05$ versus the $\mathrm{OA}+$ Allopurinol group.

performance in reducing Sua, protecting kidney function, and ameliorating renal inflammation and fibrosis. Compared with allopurinol, a classic drug for treating high levels of UA, SZF does not induce allergic reactions and is not toxic to the liver or kidneys. We delineated the therapeutic properties of SZF and its value for subsequent research and clinical applications.

\section{Conflicts of Interest}

The authors declare that they have no conflicts of interest.

\section{Authors' Contributions}

Yansheng Wu and Fei He contributed equally to this work. 


\section{Acknowledgments}

This work was supported by the National Natural Science Foundation of China (Grant no. 81373613) and Budget Project from Shanghai University of Traditional Chinese Medicine (2015YSN40). All work was performed at the TCM Institute of Kidney Disease and Shuguang Hospital, affiliated with Shanghai University of Traditional Chinese Medicine (Grant no. 14DZ2273200).

\section{References}

[1] F. Perez-Ruiz, E. Marimon, and S. P. Chinchilla, "Hyperuricaemia with deposition: latest evidence and therapeutic approach," Therapeutic Advances in Musculoskeletal Disease, vol. 7, no. 6, pp. 225-233, 2015.

[2] Y. Zhu, Y. Hu, T. Huang et al., "High uric acid directly inhibits insulin signalling and induces insulin resistance," Biochemical and Biophysical Research Communications, vol. 447, no. 4, pp. 707-714, 2014.

[3] Y. Lytvyn, B. A. Perkins, and D. Z. I. Cherney, "Uric acid as a biomarker and a therapeutic target in diabetes," Canadian Journal of Diabetes, vol. 39, no. 3, pp. 239-246, 2015.

[4] S. M. Ibrahim, E. S. El-Denshary, and D. M. Abdallah, "Geraniol, alone and in combination with pioglitazone, ameliorates fructose-induced metabolic syndrome in rats via the modulation of both inflammatory and oxidative stress status," PLOS ONE, vol. 10, no. 2, Article ID e0117516, 2015.

[5] F. Braga, S. Pasqualetti, S. Ferraro, and M. Panteghini, "Hyperuricemia as risk factor for coronary heart disease incidence and mortality in the general population: a systematic review and meta-analysis," Clinical Chemistry and Laboratory Medicine, vol. 54, no. 1, pp. 7-15, 2016.

[6] L. Sborgi, S. Rühl, E. Mulvihill et al., "GSDMD membrane pore formation constitutes the mechanism of pyroptotic cell death," EMBO Journal, vol. 35, no. 16, pp. 1766-1778, 2016.

[7] A. De Giorgi, F. Fabbian, M. Pala et al., "Uric acid: friend or foe? uric acid and cognitive function 'gout kills more wise men than simple," European Review for Medical and Pharmacological Sciences, vol. 19, no. 4, pp. 640-646, 2015.

[8] H.-Y. Chang, W.-H. Pan, W.-T. Yeh, and K.-S. Tsai, "Hyperuricemia and gout in Taiwan: results from the nutritional and health survey in Taiwan (1993-1996)," The Journal of Rheumatology, vol. 28, no. 7, pp. 1640-1646, 2001.

[9] C. Li, L. Han, A. M. Levin et al., "Multiple single nucleotide polymorphisms in the human urate transporter 1 (hURAT1) gene are associated with hyperuricaemia in Han Chinese," Journal of Medical Genetics, vol. 47, no. 3, pp. 204-210, 2010.

[10] B. Satirapoj, O. Supasyndh, N. Nata et al., "High levels of uric acid correlate with decline of glomerular filtration rate in chronic kidney disease," Journal of the Medical Association of Thailand, vol. 93, supplement 6, pp. S65-S70, 2010.

[11] M. Murea, "Advanced kidney failure and hyperuricemia," Advances in Chronic Kidney Disease, vol. 19, no. 6, pp. 419-424, 2012.

[12] Y. Isaka, Y. Takabatake, A. Takahashi, T. Saitoh, and T. Yoshimori, "Hyperuricemia-induced inflammasome and kidney diseases," Nephrology Dialysis Transplantation, vol. 31, no. 6, pp. 890-896, 2016.

[13] J. Xiao, X.-L. Zhang, C. Fu et al., "Soluble uric acid increases NALP3 inflammasome and interleukin- $1 \beta$ expression in human primary renal proximal tubule epithelial cells through the Tolllike receptor 4-mediated pathway," International Journal of Molecular Medicine, vol. 35, no. 5, pp. 1347-1354, 2015.

[14] F. Martinon, V. Pétrilli, A. Mayor, A. Tardivel, and J. Tschopp, "Gout-associated uric acid crystals activate the NALP3 inflammasome," Nature, vol. 440, no. 7081, pp. 237-241, 2006.

[15] M. Lamkanfi and V. M. Dixit, "Mechanisms and functions of inflammasomes," Cell, vol. 157, no. 5, pp. 1013-1022, 2014.

[16] R. Zhou, A. S. Yazdi, P. Menu, and J. Tschopp, "A role for mitochondria in NLRP3 inflammasome activation," Nature, vol. 469, no. 7329, pp. 221-225, 2011.

[17] A. Abderrazak, T. Syrovets, D. Couchie et al., "NLRP3 inflammasome: from a danger signal sensor to a regulatory node of oxidative stress and inflammatory diseases," Redox Biology, vol. 4, pp. 296-307, 2015.

[18] K. Labbé and M. Saleh, "Pyroptosis: A Caspase-1-Dependent Programmed Cell Death and a Barrier to Infection," in Progress in Inflammation Research, pp. 17-36, 2011.

[19] L. Yi and H. Li-qun, "Treatment of 33 cases of hyperuricemic nephropathy of phlegm-blood stasis by 'Shizhi Decoction,"' Shanghai Journal of Traditional Chinese Medicine, vol. 45, no. 9, pp. 41-43, 2011.

[20] L. B. Wang, "The effect on oxidative stress in aged uric acid nephropathy patients treated with Shizhifang," Chinese Journal of Gerontology, vol. 36, no. 18, pp. 4556-4558, 2016.

[21] M. Mazzali, J. Hughes, Y.-G. Kim et al., "Elevated uric acid increases blood pressure in the rat by a novel crystal-independent mechanism," Hypertension, vol. 38, no. 5, pp. 1101-1106, 2001.

[22] M.-X. Wang, Y.-L. Liu, Y. Yang, D.-M. Zhang, and L.-D. Kong, "Nuciferine restores potassium oxonate-induced hyperuricemia and kidney inflammation in mice," European Journal of Pharmacology, vol. 747, pp. 59-70, 2015.

[23] S. Joshi, W. Wang, A. B. Peck, and S. R. Khan, "Activation of the NLRP3 inflammasome in association with calcium oxalate crystal induced reactive oxygen species in kidneys," The Journal of Urology, vol. 193, no. 5, pp. 1684-1691, 2015.

[24] A. Ives, J. Nomura, F. Martinon et al., "Xanthine oxidoreductase regulates macrophage IL1 $\beta$ secretion upon NLRP3 inflammasome activation," Nature Communications, vol. 6, article 6555, 2015.

[25] C.-Y. Fan, M.-X. Wang, C.-X. Ge, X. Wang, J.-M. Li, and L.-D. Kong, "Betaine supplementation protects against highfructose-induced renal injury in rats," The Journal of Nutritional Biochemistry, vol. 25, no. 3, pp. 353-362, 2014.

[26] M. N. Darisipudi and F. Knauf, "An update on the role of the inflammasomes in the pathogenesis of kidney diseases," Pediatric Nephrology, vol. 31, no. 4, pp. 535-544, 2016.

[27] M. Cristóbal-García, F. E. García-Arroyo, E. Tapia et al., "Renal oxidative stress induced by long-term hyperuricemia alters mitochondrial function and maintains systemic hypertension," Oxidative Medicine and Cellular Longevity, vol. 2015, Article ID 535686, 8 pages, 2015.

[28] S. Park, J.-H. Won, I. Hwang, S. Hong, H. K. Lee, and J.-W. $\mathrm{Yu}$, "Defective mitochondrial fission augments NLRP3 inflammasome activation," Scientific Reports, vol. 5, Article ID 15489, 2015.

[29] K. L. Rock, H. Kataoka, and J.-J. Lai, "Uric acid as a danger signal in gout and its comorbidities," Nature Reviews Rheumatology, vol. 9, no. 1, pp. 13-23, 2013.

[30] E. Yoshihara, S. Masaki, Y. Matsuo, Z. Chen, H. Tian, and J. Yodoi, "Thioredoxin/txnip: redoxisome, as a redox switch for 
the pathogenesis of diseases," Frontiers in Immunology, vol. 4, article 514, 2014.

[31] X. Zhang, J.-H. Zhang, X.-Y. Chen et al., "Reactive oxygen species-induced TXNIP drives fructose-mediated hepatic inflammation and lipid accumulation through NLRP3 inflammasome activatio," Antioxidants \& Redox Signaling, vol. 22, no. 10, pp. 848-870, 2015.

[32] E.-S. Ryu, M. J. Kim, H.-S. Shin et al., "Uric acid-induced phenotypic transition of renal tubular cells as a novel mechanism of chronic kidney disease," American Journal of Physiology-Renal Physiology, vol. 304, no. 5, pp. F471-F480, 2013.

[33] C. Wang, Y. Pan, Q.-Y. Zhang, F.-M. Wang, and L.-D. Kong, "Quercetin and allopurinol ameliorate kidney injury in STZtreated rats with regulation of renal NLRP3 inflammasome activation and lipid accumulation," PLoS ONE, vol. 7, no. 6, Article ID e38285, 2012.

[34] J. Shi, Y. Zhao, K. Wang et al., "Cleavage of GSDMD by inflammatory caspases determines pyroptotic cell death," Nature, vol. 526, no. 7575, pp. 660-665, 2015.

[35] N. Kayagaki, I. B. Stowe, B. L. Lee et al., "Caspase-11 cleaves gasdermin D for non-canonical inflammasome signalling," Nature, vol. 526, no. 7575, pp. 666-671, 2015.

[36] P. Broz, "Immunology: caspase target drives pyroptosis," Nature, vol. 526, no. 7575, pp. 642-643, 2015.

[37] H. Chen, Y. Lu, Z. Cao et al., "Cadmium induces NLRP3 inflammasome-dependent pyroptosis in vascular endothelial cells," Toxicology Letters, vol. 246, pp. 7-16, 2016.

[38] J.-R. Yang, F.-H. Yao, J.-G. Zhang et al., "Ischemia-reperfusion induces renal tubule pyroptosis via the CHOP-caspase-11 pathway," American Journal of Physiology-Renal Physiology, vol. 306, no. 1, pp. F75-F84, 2014.

[39] S. D. Chung, T. Y. Lai, C. T. Chien, and H. J. Yu, "Activating Nrf-2 signaling depresses unilateral ureteral obstructionevoked mitochondrial stress-related autophagy, apoptosis and pyroptosis in kidney," PLoS ONE, vol. 7, no. 10, Article ID e47299, 2012. 


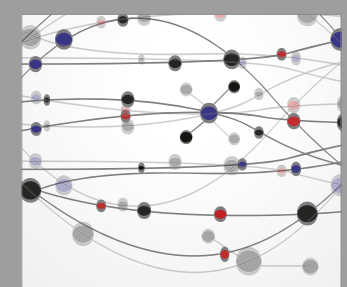

The Scientific World Journal
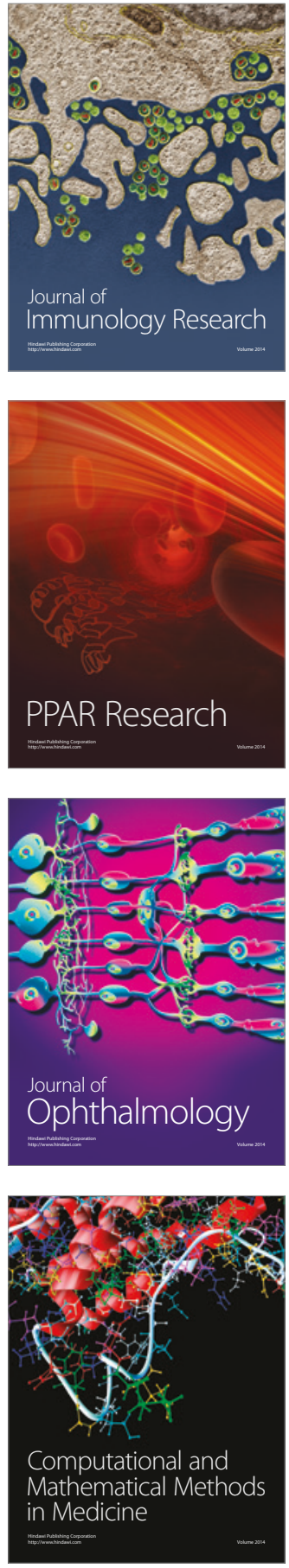

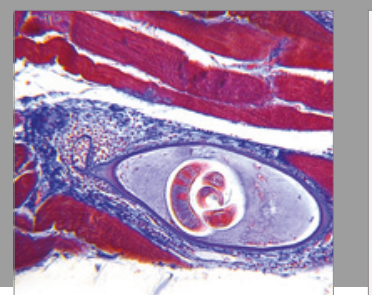

Gastroenterology Research and Practice
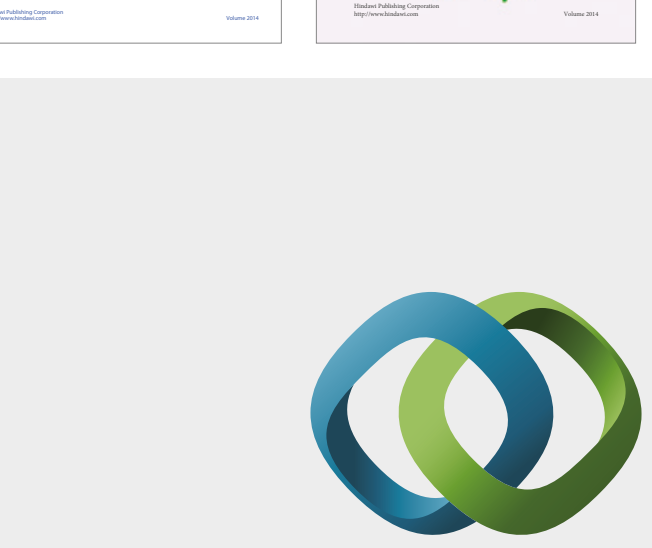

\section{Hindawi}

Submit your manuscripts at

https://www.hindawi.com
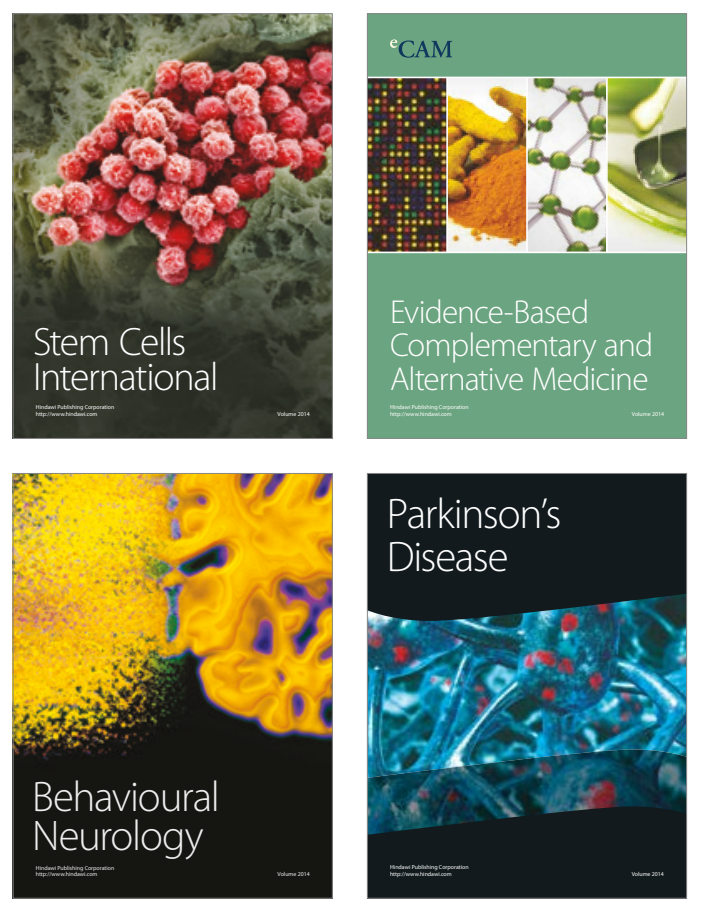
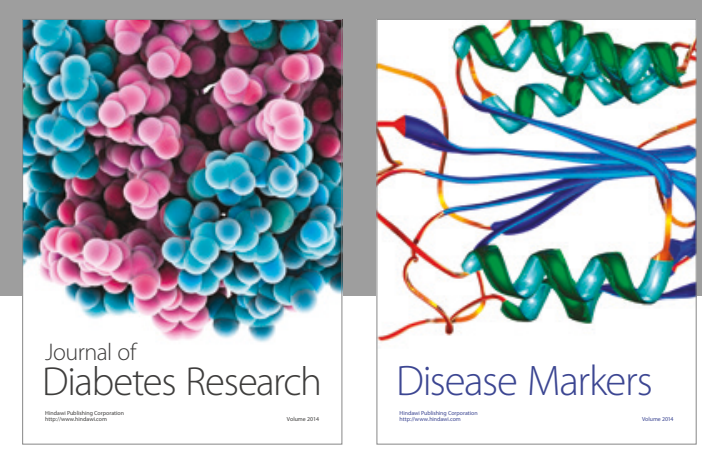

Disease Markers
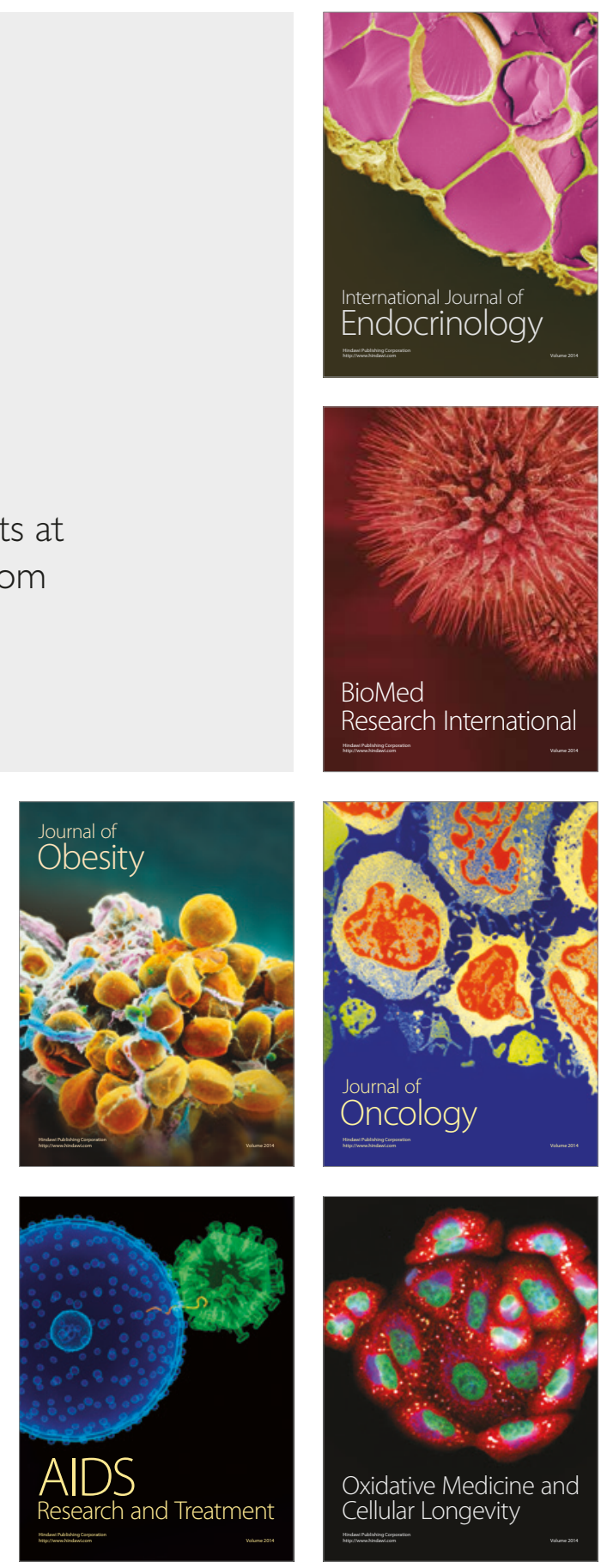